\title{
Government Formation in a Two Dimensional Policy Space*
}

\author{
Enriqueta Aragonès ${ }^{\dagger}$ \\ Institut d'Anàlisi Econòmica, CSIC
}

April 2006

\begin{abstract}
Given any allocation of parliament seats among parties, we characterize all the stable government configurations (supported by at least a majority of the parliament) in terms of winning coalitions and policy outcomes. We consider a two dimensional policy space and we assume that there are four parties that care mainly about holding office, and only instrumentally about policy. We find that for any distribution of seats in the parliament only two scenarios are possible: either there is a party that is a member of almost all equilibrium coalitions (dominant party scenario) or there is a party that is never a member of an equilibrium coalition (dominated party scenario). We characterize the key party for each possible scenario and we show that it is sufficient that the key party has intense preferences over one the issues to guarantee the formation of a stable government coalition.
\end{abstract}

\section{Introduction}

In this paper we analyze a bargaining model of government formation in parliamentary democracies. We try to understand how a given electoral result leads to the formation of a governing coalition. Our predictions are described by a coalition of parties supported by a majority of votes, and a policy supported by the parties in the coalition. We characterize all stable government configurations in terms of coalitions and policies.

${ }^{*}$ I am grateful to Itzhak Gilboa and an anonymous referee for detailed comments on an earlier draft. I gratefully acknowledge financial support from the Spanish Ministry of Science and Technology, grant number SEC2003-01961 and CREA-Barcelona Economics. This paper is part of the Polarization and Conflict Project CIT-2-CT-2004-506084 funded by the European Commission-DG Research Sixth Framework Programme. This article reflects only the author's views and the Community is not liable for any use that may be made of the information contained therein.

†Institut d'Anàlisi Econòmica, CSIC. Campus UAB, 08193 Bellaterra (Spain). enriqueta.aragones@uab.cat 
There are different spatial models of legislative choice that seek to describe or predict how individual legislators make collective choices, and the model we present belongs to this literature. Our contribution is an extension of the analysis of government formation in a parliamentary democracy to a multidimensional policy space. Krehbiel (1988) states that "expanding the dimensionality of the choice space from one to two has profoundly disequilibrating consequences." The main problem with a two dimensional space in a voting game is the potential for voting cycles and chaos, as predicted by McKelvey's (1976) chaos theorem.

Following an important part of the literature we will use the concept of the core, borrowed from cooperative game theory, as a measure of stability (see, for instance, Moulin (1988) or Myerson (1990)). This assumption is particularly relevant when we consider the formation of the coalition that sustains an executive in office on the basis of a confidence vote. The members of this coalition consume all the benefits of office-holding, and have the control of all policy outputs. There is no binding agreement in this coalition: a non-confidence motion may be proposed at any time.

Plott (1967) describes the particular conditions under which there is a nonempty 'core' in two dimensional voting games. In the legislative bargaining model that we present the non-empty core conditions are satisfied for a large range of parameter values. These conditions guarantee the formation of a stable governing coalition.

We consider purely opportunistic parties, that is, parties that value their office holding in the present and in future legislatures. We assume that voters are ideological, that is, they care about the policies implemented. When facing ideological voters, parties that care about holding office today and also about holding office in future legislatures, may decide not to accept a proposal to join a governing coalition if it implies the implementation of a policy that is too different from their ideal point. The reason is that by compromising their policy position in the present they might jeopardize their vote support in future elections. That is, parties will be concerned with their policy positions only to the extend that this helps them to win elections.

We represent the parties payoffs in a reduced form by assuming that parties care about holding office and instrumentally about the policy implemented. The value that a party attaches to holding office today, that is, how much they are willing to give up on policy preferences today, can be though as determined by the party preferences for holding office in the future. Alternatively, one can think of the value for holding office as an individual rationality constraint for the party: it represents the maximal amount of utility that it is willing to give up in terms of policy. A party that attaches a large value to holding office will be willing to commit to policies far away from its ideal point in order to guarantee becoming a member of the winning coalition.

We assume complete party discipline, that is, we assume that all members of a party share the same policy preferences. Therefore, we define the preferences and actions of a given party as representing the preferences and actions of all its members. 
We focus our analysis on a two dimensional policy space and we assume that there are four parties. We endow these parties with flexible relative intensity of preferences between issues: their indifference curves with respect to policies are not circles but rather ellipses.

Our analysis is mainly concerned with the parties' negotiations in order to form a governing coalition and to determine a policy outcome. Thus, we do not model the electoral competition stage, we only take as given an electoral result and its corresponding parliamentary representation. We assume that a governing coalition can only be formed with the support of a majority of the votes of the parliament, that is, it must be a winning coalition according to majority rule. And we also assume that only governing coalitions can decide on the policy to be implemented. In case a single party has a majority, it can implement its ideal point on both issues. Our interest focuses on those cases in which no party has a majority.

Since policies can only be implemented by a governing coalition, voters consider that only the parties that are members of the governing coalition are responsible of the policy choice. Thus, we assume that the payoffs of those parties that are not members of the government are not affected by the policy implemented.

Given any allocation of parliament seats among parties, we characterize all the stable government configurations in terms of winning coalitions and policy outcomes. Our analysis shows that, for any given allocation of parliament seats among parties, there are only two possible kinds of scenarios: either there is a party that may form a winning coalition with any other party (dominant party scenario), or there is a party that is not a member of any winning coalition (dominated party scenario). We characterize the key party for each possible scenario and we show that it is sufficient that the key party has intense preferences over one the issues to guarantee the formation of a stable government coalition.

The rest of the paper is organized as follows: the next section explains where this paper fits in the existing literature, section 3 describes the formal model and the equilibrium concept, section 4 characterizes the coalitions that are equilibrium candidates, and section 5 characterizes the policies that can be supported in equilibrium by such coalitions. Section 6 describes the equilibrium outcomes, and section 7 contains some concluding remarks.

\section{Related Literature}

In many of the existing models it is assumed that parties are office-seeking, that is, their payoffs depend only on whether they are members of the winning coalition. Some of them consider policy blind theories, and model coalition bargaining as a constant sum game. In this line we find the Minimal Winning Coalition Theory by von Neumann and Morgenstern (1953), the Minimum Winning Coalition Theory by Riker (1962), and Leiserson's (1966) refinement of the Minimal Winning Coalition Theory using the 'smallest number of parties' 
bargaining principle. Other models assume that policy choice plays a role in the parties' payoffs. In some of these models policy is assumed to be an aid to bargain, such as the Minimal Connected Winning Coalition Theory by Axelrod (1970), and its refinement based on the smallest ideological range by de Swaan (1973).

The assumption that we make on parties' objective function is similar to the one made by Austen-Smith and Banks (1988), where bargaining over policy is a form of electoral competition. They assume that voters are policy motivated, and choose their vote with the aim to affect the policy outcome. They also assume that voters can forecast the coalition that would form following each possible election outcome and they can calculate back from this forecast to decide how best to cast their vote. Our model can be thought of as a reduced form game of their model of repeated elections, where we take as given the reaction of the voters, and we extend it over a two dimensional policy space.

The theoretical work on coalition formation that assume more than one policy dimension has to solve the problem of voting cycles and chaos. Some of these models incorporate particular legislative structures, for example: Shepsle's (1979) structure induced equilibrium, Baron and Ferejohn (1987) on distributive 'pork barrel', and its extension by Jackson and Moselle (2002) that includes an ideological decision. Other models introduce uncertainty and imperfect information, as in Enelow and Hinich (1983) or consider the role of party discipline, as in McKelvey and Schofield (1987). Most of these works are concerned with legislative politics in the US and they are ill-suited to explain the formation of government coalitions in a parliamentary democracy.

Most of the work on the politics of coalition in parliamentary democracies has been empirical, seeking to account for the coalitions that actually form. A comprehensive survey can be found in Laver and Schofield (1990). Diermeier, Eraslan and Merlo (2003) set up a model of government formation in order to test the relationship of different feature to a measure of its stability. And Diermeier and Merlo (2004) estimate how the selection of the formateur affects the formation of stable governments.

\section{The Model}

We consider a two-dimensional policy space represented by $\Re^{2}$. We denote a policy by $(x, y) \in \Re^{2}$ and we interpret $x$ and $y$ as positions on the two issues, $X$ and $Y$. Parties are characterized by their ideal points in the policy space and by the value that they attach to holding office. Let $\left(x_{i}, y_{i}\right) \in \Re^{2}$ denote the ideal point of party $i$. There are four parties denoted by $i=00,01,10$, and 11 , whose ideal points are $(0,0),(0,1),(1,0)$, and $(1,1)$ respectively.

We assume that an election has already taken place, and the proportions of parliament seats that each party has obtained are given by $v_{00}, v_{01}, v_{11}$, and $v_{10}$ for parties $00,01,11$, and 10 respectively. We assume that $v_{00}+v_{01}+v_{11}+v_{10}=1$ and $0 \leq v_{i}<\frac{1}{2}$ for all $i \in\{00,01,11,10\}$. For simplicity we rule out the case $v_{i}=\frac{1}{2}$. Indeed, many parliaments have an odd number of seats. And we do not 
consider the possibility that a party has a majority of the seats, that is, $v_{i}>\frac{1}{2}$ for some party $i$, since the analysis of this case would lead to trivial results.

We assume that parties are mainly concerned about holding office, that is, being members of the governing coalition. We assume that only parties that are members of the governing coalition care about the policy implemented, since it may affect their vote support in future elections. We assume that the payoff of parties that are not members of the governing coalition is not affected by the policy choice of the government. Thus, we normalize the utility of a party that is not a member of the governing coalition to zero, and we represent the utility that party $i$ obtains if it becomes a member of the governing coalition when the implemented policy is $(x, y) \in \Re^{2}$ by $U_{i}(x, y)$. Therefore, the payoff function of party $i$ can be defined as follows:

$$
V_{i}(C,(x, y))=\left\{\begin{array}{ccc}
0 & \text { if } & i \notin C \\
U_{i}(x, y) & \text { if } & i \in C
\end{array}\right.
$$

Where $C$ denotes a winning coalition. Since we assume that no party obtains a majority of the votes, parties are supposed to form coalitions and negotiate over the policy to be implemented.

We represent by $k_{i} \in \Re^{+}$the utility that a party derives from being a member of the governing coalition and we assume that the utility that party $i$ obtains if it becomes a member of the governing coalition when the implemented policy is $(x, y) \in \Re^{2}$ is given by:

$$
U_{i}(x, y)=k_{i}-a_{i}\left(x-x_{i}\right)^{2}-b_{i}\left(y-y_{i}\right)^{2} \text { with } a_{i}, b_{i}>0 \text { and } a_{i}+b_{i}=1 \text {. }
$$

According to this utility function, parties' preferences over policies are single peaked, convex but not necessarily symmetric. The parameters $a_{i}$ and $b_{i}$ represent the relative importance of the issues in the ideology of party $i$ (see figure 1). If $a_{i}=b_{i}$, both issues have the same effect on the utility of party $i$, thus both issues are as important in the ideology of party $i$. If $a_{i}>b_{i}$ the first issue is regarded as more important than the second by party $i$, and if $a_{i}<b_{i}$ the second issue is regarded as more important than the first. We rule out the possibility that $a_{i}=0$ or $a_{i}=1$, since we are only interested in cases in which a party cares about more than one issue. Since $b_{i}=1-a_{i}$, we have that as the value of $a_{i}$ increases, the first issue becomes more important for party $i$, and therefore party $i$ requires a more favorable compromise on the second issue for a given deal on the first.

Notice that the utility that a party obtains from becoming a member of the governing coalition, $k_{i}$, may also be thought of as a reservation value: a party will never accept to become a member of a governing coalition if it has to support a policy that gives it a (dis)utility larger than its value of holding office. Thus, the value of holding office defines an Individual Rationality constraint for the party. Formally, the set of policies from which party $i$ derives a utility of zero, defines the boundary of the set of policies that are Individually Rational 
for party $i, A_{i}=\left\{(x, y): U_{i}(x, y) \geq 0\right\}$. The size of this set depends on the magnitude of $k_{i}$ : the larger the value of holding office the larger the set of policies that party $i$ is willing to support in a given governing coalition. That is, the more a party values to be a member of the governing coalition the more flexible will it be in terms of trading-off policy.

Within this framework we define an equilibrium outcome as a winning coalition and a policy compromise that induce a utility distribution that satisfies the stand-alone principle. This implies that there is no other winning coalition that could stand-alone with a policy that would improve the welfare of all its members.

Definition: An equilibrium outcome is a coalition of parties $C^{*}$, and a policy $\left(x^{*}, y^{*}\right) \in \Re^{2}$ such that:

1) $C^{*}$ is a winning coalition.

2) There is no other $(C,(x, y))$ such that $C$ is a winning coalition and $V_{i}(C,(x, y)) \geq V_{i}\left(C^{*},\left(x^{*}, y^{*}\right)\right)$ for all $i \in C$, with at least one strict inequality.

From the assumptions of the model and the definition of equilibrium we obtain the following preliminary results:

1) The subset of the policy space that is relevant for the negotiations among parties is the Pareto set, which in our case is $[0,1]^{2}$ (see figure 2 ).

2) The policies selected as equilibrium outcomes will be a subset of the policies that are Pareto Optimal and Individually Rational for all coalition members.

3) Generically, equilibrium coalitions must be minimal winning coalitions.

The first two results are directly implied by the assumptions of the model. The last one requires an additional explanation. A minimal winning coalition is defined as a winning coalition that would not be supported by a majority of votes if one of its members was removed from it. Here we have that for any policy outcome supported by a winning coalition that is not minimal there is a policy outcome supported by a corresponding minimal winning coalition, that is considered at least as good by its coalition members. Thus, the standalone principle implies that generically we will have that only minimal winning coalitions can be part of an equilibrium outcome, as predicted by von Neumann and Morgenstern (1953).

Non generically, for some policies that are supported by a winning coalition that is not a minimal winning coalition the corresponding indifference curves for two of the parties may be tangent. In this case, if these two same parties are the ones that form a winning coalition, the larger coalition may be part of an equilibrium. But in this case, we will have that the equilibrium policies supported by both coalitions coincide. Therefore, without loss of generality, we will restrict our equilibrium analysis to minimal winning coalitions. 


\section{Winning Coalitions}

Given the proportion of seats of each party, $v_{00}, v_{01}, v_{11}$, and $v_{10}$, we analyze the different possible governing coalitions. From the preliminary results stated in the previous section, we restrict our attention to minimal winning coalitions. We characterize the different possible scenarios scenarios in terms of the number and structure of the minimal winning coalitions that are candidates for equilibrium coalitions. Since we have assumed that $v_{00}+v_{01}+v_{11}+v_{10}=1$ and $0 \leq v_{i}<\frac{1}{2}$ for all $i \in\{00,01,11,10\}$, we may state the following results:

\section{Proposition 1:}

1) All winning coalitions of two parties are minimal winning coalitions.

2) There are always three minimal winning coalitions of two parties.

3) All coalitions of three parties are winning coalitions.

4) There is at most one minimal winning coalition of three parties.

All proofs can be found in the Appendix. Since only minimal winning coalitions may be part of an equilibrium, given the previous results, we have only two possible different scenarios in equilibrium: either there is a three party minimal winning coalition or there is none. In the first case, which we denote the "dominant party scenario", the party that is not a member of the minimal winning coalition of three parties must be a member of all winning coalitions of two parties. In the second case, which we denote the "dominated party scenario", there is a party that is never a member of a minimal winning coalition.

\section{Proposition 2:}

i) There is a minimal winning coalition of three parties if and only if there is a party that is a member of all winning coalitions of two parties.

ii) There is no minimal winning coalition of three parties if and only if there is a party that is never a member of a minimal winning coalition.

Observe that in the dominant party scenario, there are four minimal winning coalitions: a minimal winning coalition of three parties that involves all parties except the dominant one, and three winning coalitions of two parties, formed by the dominant party plus each one of the other three parties. In our set up, each party is always a member of three coalitions of two parties. In the dominant party scenario, the three coalitions of two parties that include the dominant party are winning coalitions, and for each one of the other parties only one of the two party coalitions they belong to is a winning coalition.

In the dominated party scenario there are three minimal winning coalitions: all of them are formed by two parties. The dominated party is not a member of any of them. Each of the other parties are members of exactly two winning coalitions of two parties.

A different way to characterize the two possible scenarios is by considering the relative size of the proportion of seats by the parties. Suppose that we order the four parties according to the number of parliament seats each one controls 
and denote them accordingly, that is, $v_{1} \geq v_{2} \geq v_{3} \geq v_{4}$. We have to consider four cases:

i) $v_{1}>\frac{1}{4}>v_{2} \geq v_{3} \geq v_{4}$.

ii) $v_{1}>v_{2}>\frac{1}{4}>v_{3}>v_{4}$ and $v_{1}+v_{4}>\frac{1}{2}$

In these cases we are in the dominant party scenario. The party with the largest number of seats is the dominant party.

iii) $v_{1} \geq v_{2} \geq v_{3}>\frac{1}{4}>v_{4}$

iv) $v_{1}>v_{2}>\frac{1}{4}>v_{3}>v_{4}$ and $v_{1}+v_{4}<\frac{1}{2}$

In these cases we are in the dominated party scenario. The party with the smallest number of seats is the dominated party.

Notice that since we have assumed an odd number of seats in the parliament, no party can ever obtain exactly one fourth of the proportion of seats, and no coalition of two parties can ever obtain exactly one half of the seats.

Finally, observe that in order to be in the dominant party scenario there must be at least two parties that control more than one fourth of the seats, while in order to be in the dominated party scenario there must be at most two parties that control more than one fourth of the seats.

\section{$5 \quad$ Acceptable Policies}

A winning coalition of two parties faces a bargaining problem. A policy will be acceptable by the coalition if it gives both parties a utility level of at least their reservation value and it is Pareto Optimal within its bargaining set, that is, there is no other policy that gives a larger utility to one of the parties and no smaller to the other one, and satisfies Individual Rationality for both. We define the set of policies that are acceptable by a coalition of two parties, $i$ and $j$, by $A(i, j)$. We show under which conditions this set is not empty and under which conditions it does not contain the parties' ideal points. First, we analyze the sets of acceptable policies by coalitions of parties that agree on one of the issues.

Proposition 3: Consider a coalition of two parties, $i$ and $j$, that agree on issue $x$ at $x=\bar{x}$.

i) $A(i, j)=\left\{(\bar{x}, y) \in[0,1]^{2}: 1-\sqrt{\frac{k_{i}}{b_{i}}} \leq y \leq \sqrt{\frac{k_{j}}{b_{j}}}\right\}$

ii) $A(i, j)$ is not empty if and only if $1 \leq \sqrt{\frac{k_{i}}{b_{i}}}+\sqrt{\frac{k_{j}}{b_{j}}}$.

iii) $A(i, j)$ does not include party $i$ 's ideal point if and only if $k_{j}<b_{j}$.

iv) $A(i, j)$ does not include party $j$ 's ideal point if and only if $k_{i}<b_{i}$.

This proposition shows that the set of policies that are acceptable by two parties that agree on issue $x$ is larger, the larger is the parties' preference intensity for issue $x$, that is, the smaller $b_{i}$. The intuition is clear: two parties that agree on one of the issues and care a lot more about this issue than the rest, will have a lot of policies that would be preferred by both to their reservation value. 
Notice that the set of policies acceptable by a given coalition is larger, the larger are the parties' values of holding office, that is, the less restrictive is the condition of individual rationality. On the other hand, the larger are the parties' values of holding office, the more likely it is that their ideal points are included in the set of acceptable policies.

See figure 3. A similar argument can be applied to characterize the set of acceptable policies by any coalition of two parties that agree on issue $y$.

Next, we analyze the sets of acceptable policies by coalitions of parties that disagree on both issues but have the same relative intensity of preferences.

Proposition 4: Consider a coalition of two parties, $i$ and $j$, that disagree on both issues, and suppose $a_{i}=a_{j}$ :

i) $A(i, j)$ is not empty for all $a_{i}=a_{j}$ if and only if $1 \leq \sqrt{k_{i}}+\sqrt{k_{j}}$.

ii) $A(i, j)$ does not include party $i$ 's ideal point if and only if $k_{j}<1$.

iii) $A(i, j)$ does not include party $j$ 's ideal point if and only if $k_{i}<1$.

This proposition also provides a full characterization of the set of policies that are acceptable by these coalitions, and the conditions under which these sets are not empty and the bargaining within the coalition is not trivial.

The intuition in this case is similar to the one encountered before: the set of policies acceptable by a given coalition is larger, the larger are the parties' values of holding office, that is, the less restrictive is the condition of individual rationality. On the other hand, the larger are the parties' values of holding office, the more likely it is that their ideal points are included in the set of acceptable policies.

Finally, we analyze the sets of acceptable policies by coalitions of parties that disagree on both issues but have different relative intensity of preferences. Consider the coalition of two parties that disagree on both issues $00-11$, and suppose that $a_{00} \neq a_{11}$. In this case the Pareto optimal policies within the bargaining set of this coalition are the solution to the following maximization problem:

$$
\begin{gathered}
\max k_{11}-a_{11}(1-x)^{2}-b_{11}(1-y)^{2} \\
\text { s.t. } k_{00}-a_{00}(x)^{2}-b_{00}(y)^{2} \geq u_{00}
\end{gathered}
$$

From the first order conditions we obtain $\frac{a_{11}(1-x)}{b_{11}(1-y)}=\frac{a_{00} x}{b_{00} y}$. Notice that in general, if $a_{i} \neq a_{j}$ the contract curve between two parties that disagree on both issues is not a straight line (as it was when we assumed that $a_{i}=a_{j}$ ). Now the contract curve is a concave or convex line depending on whether $a_{i}$ is smaller or larger than $a_{j}$ (See figure 4).

Even though we cannot fully characterize the set of acceptable policies in this case, we know that it has to be a segment of the contract curve of the coalition determined by the Individual rationality constraints, and we have an analytical characterization of this curve (See figures 5 and 6 ). Furthermore, we can obtain a sufficient condition for this set not to be empty. 
Proposition 5: Consider a coalition of two parties, $i$ and $j$, that disagree on both issues and suppose $a_{i} \neq a_{j}$ :

i) $A(i, j)$ is not empty for any $a_{i}$ and $a_{j}$ if $k_{i}>\frac{1}{4}$ and $k_{j}>\frac{1}{4}$.

ii) $A(i, j)$ does not include party $i$ 's ideal point if and only if $k_{j}<1$.

iii) $A(i, j)$ does not include party $j$ 's ideal point if and only if $k_{i}<1$.

Similarly, a winning coalition of three parties also faces a bargaining problem. A policy will be acceptable by the coalition if it gives to all parties in the coalition a utility level of at least their reservation value and it is Pareto Optimal within its bargaining set.

Consider coalition $01-11-10$. The set of policies that satisfy the Individual Rationality conditions for all three parties are the pairs $(x, y)$ that satisfy the following three conditions:

$$
\begin{aligned}
& k_{01}-a_{01}(x)^{2}-b_{01}(1-y)^{2} \geq 0 \\
& k_{10}-a_{10}(1-x)^{2}-b_{10}(y)^{2} \geq 0 \\
& k_{11}-a_{11}(1-x)^{2}-b_{11}(1-y)^{2} \geq 0
\end{aligned}
$$

Notice that with respect to the set of policies that satisfy Individual Rationality for all three parties, all policies are also Pareto optimal. Therefore, these conditions define the set of acceptable policies by the coalition of three parties. See figures 7 and 8 . Let $A(01,11,10)$ denote this set.

For this set to be non-empty it is necessary that the conditions stated in the propositions of the previous section hold, that is, it is necessary that the set of acceptable policies of each coalition of two parties involved in the three party coalition are not empty. And in addition, it is also necessary that these sets have a non empty intersection.

Therefore, the set of policies that are acceptable by a coalition of three parties is not empty if for all parties $i$ in the coalition, $k_{i}$ are large enough.

That is, the sets of policies that are individually rational for each one of the coalition members are large enough so that their intersection is not empty. The conditions that guarantee a non trivial bargaining within the members of the coalition, are not restrictive in this case.

Observe that, given the utility functions of the parties, the set of policies that are acceptable by a coalition of three parties, when it is not empty, is a compact set and the policy that maximizes the utility of a given party within this set is well defined and it is unique. Let $\left(x_{i}(01-11-10), y_{i}(01-11-10)\right)$ denote the policy that maximizes the utility of party $i$ within the set of acceptable policies of the three party coalition $01-11-10$, for some $i \in\{01,11,10\}$. This implies that $U_{j}\left(x_{i}(01-11-10), y_{i}(01-11-10)\right)=0$ for at least one party $j$ in the coalition with $j \neq i$. In particular, for party 11 we have that for all $j \neq 11, U_{j}\left(x_{11}(01-11-10), y_{11}(01-11-10)\right)=0$.

This analysis provides a full characterization of the set of policies that are acceptable by the coalitions, and conditions under which these sets are not empty. These conditions involve restrictions on the range of parameter values and they have to be satisfied by any party that is part of an equilibrium coalition. Finally, it provides conditions under which these sets do not include the parties' ideal points. We are going to impose these conditions in order to guarantee 
bargaining among the parties in a coalition is not trivial. Thus, the combination of these conditions implies that we will analyze the bargaining among parties whose value of holding office is not too large, and not too small.

\section{Equilibrium Outcomes}

In this section we assume that the set of acceptable policies for each one of the relevant winning coalitions, $A(C)$, is not empty and it does not contain any of the ideal points of its members. This assumption only imposes restrictions on the possible values of $k_{i}$ for all $i$, that is, the value of holding office for each party is large enough so that the set of policies that it finds acceptable intersects with each one of the other parties' set of acceptable policies; and it is small enough so that these intersections do not include the parties ideal points.

We find that, given a set of parameter values $\left(k_{i}, v_{i}, a_{i}\right)$ for $i \in\{00,01,11,10\}$, generically the equilibrium outcome is unique in terms of equilibrium coalitions. To prove this claim suppose that for a given set of parameter values we have two equilibrium outcomes $\left(C^{*},\left(x^{*}, y^{*}\right)\right)$ and $\left(C^{*^{\prime}},\left(x^{*^{\prime}}, y^{*^{\prime}}\right)\right)$ then:

1) If $C^{*}$ and $C^{*^{\prime}}$ have an empty intersection, then they are complements to each other, and we know that the complement of a winning coalition is a losing coalition. Thus, they cannot be both part of equilibrium outcomes.

2) If $C^{*}$ and $C^{*^{\prime}}$ have a non empty intersection, then generically a party member of the intersection would prefer one policy outcome to the other one, in which case the outcome corresponding to the least preferred policy could not be an equilibrium.

Thus, in order to have multiple equilibria there has to be a party $i$ that is a member of both $C^{*}$ and $C^{*^{\prime}}$ and in addition this party must be indifferent between $\left(x^{*}, y^{*}\right)$ and $\left(x^{*^{\prime}}, y^{*^{\prime}}\right)$, which can only happen generically.

\subsection{Dominated Party Scenario}

In order to characterize the equilibrium outcomes in the "dominated party scenario" without loss of generality we consider a particular case in which the dominated party is party 10 and the minimal winning coalitions are given by: $00-01,00-11$, and $01-11$.

The relevant strategies in this scenario are the following: party 00 may accept to form a coalition with either party 01 or party 11; party 01 may accept to form a coalition with either party 00 or party 11 ; finally, party 11 may accept to form a coalition with either party 00 or party 01 . We find that coalition $00-01$ forms in equilibrium if and only if party 01's relative preference intensity for issue $x$ is large enough. Similarly, we can prove that coalition $01-11$ forms in equilibrium if and only if party 01's relative preference intensity for issue $x$ is small enough. Otherwise, existence of equilibrium is only guaranteed under certain conditions and when they hold coalition $00-11$ forms.

\section{Theorem 1:}


In the "dominated party scenario" when $a_{00}=a_{11}$ :

i) Coalition $00-01$ forms in equilibrium if and only if $a_{01} \geq \overline{a_{01}}$

ii) Coalition $01-11$ forms in equilibrium if and only if $a_{01} \leq \underline{a_{01}}$

iii) Coalition 00-11 forms in equilibrium if and only if $1 \leq \sqrt{a_{11}}\left(1-\sqrt{\frac{k_{01}}{a_{01}}}\right)+$ $\sqrt{b_{00}}\left(1-\sqrt{\frac{k_{01}}{b_{01}}}\right)$

The two thresholds are given by: $\overline{a_{01}}=\frac{1}{1+\left(\frac{1-\sqrt{\frac{k_{11}}{a_{11}}}}{1-\frac{1-\sqrt{k_{11}}}{\sqrt{b_{00}}}}\right)^{2}}$ and

$$
\frac{a_{01}}{1+\left(\frac{1-\frac{1-\sqrt{k_{00}}}{\sqrt{a_{11}}}}{1-\sqrt{\frac{k_{00}}{b_{00}}}}\right)^{2}} \text {. }
$$

This theorem characterizes the parameter values for which an equilibrium exists. Otherwise, we obtain cycles in the negotiations between the parties, in which case the governing coalitions that may form would be unstable. For $a_{00}=a_{11}$ we have characterized the equilibrium outcomes for the dominated party scenario according to the values of $a_{01}$. We find that for values of $a_{01}$ small enough, the unique equilibrium coalition is $01-11$ and the set of equilibrium policies is given by

$$
A^{*}(01,11)=\left\{(x, 1) \in[0,1]^{2}: 1-\frac{1-\sqrt{k_{00}}}{\sqrt{a_{11}}} \leq x \leq \sqrt{\frac{b_{01}}{a_{01}}}\left(1-\sqrt{\frac{k_{00}}{b_{00}}}\right)\right\}
$$

The range of values of $a_{01}$ for which coalition 01-11 is part of an equilibrium outcome is larger if $k_{00}$ and $a_{11}$ are smaller and $b_{00}$ is larger (see figure 9).

Similarly, for values of $a_{01}$ large enough, the unique equilibrium coalition is $01-00$ and the set of equilibrium policies is given by

$$
A^{*}(00,01)=\left\{(0, y) \in[0,1]^{2}: 1-\sqrt{\frac{a_{01}}{b_{01}}}\left(1-\sqrt{\frac{k_{11}}{a_{11}}}\right) \leq y \leq \frac{1-\sqrt{k_{11}}}{\sqrt{b_{00}}}\right\}
$$

The range of values of $a_{01}$ for which coalition $01-00$ is part of an equilibrium outcome is larger if $k_{11}$ and $b_{00}$ are smaller and $a_{11}$ is larger (see figure 10).

For intermediate values of $a_{01}$, coalition $00-11$ may form in equilibrium only if $a_{00}$ and $b_{11}$ are large enough and $k_{01}$ is small enough (see figure 11).

We have shown that the parameter value that determines which equilibrium prevails in this case is $a_{01}$ (see figure 12). Observe that the conditions for existence of equilibria with governing coalitions $01-00$ and $01-11$ are much weaker that those needed for the equilibria with governing coalition $00-11$. Thus, it seems most likely that the decision of party 01 will determine which governing coalition forms when party 10 is a dominated party. Therefore, even if party 01 is not a dominant party it has the key to determine the government composition. We will call this party the 'key party' of the 'dominated party scenario. Notice that the key party in this scenario is always characterized by disagreeing on all issues with the dominated party. Finally, observe that the intuition behind this result carries over to the case of $a_{00} \neq a_{11}$, and the qualitative results obtained here can be reproduced in the more general case. 


\subsection{Dominant Party Scenario}

In order to analyze the equilibrium outcomes in the "dominant party scenario" without loss of generality we consider a particular case in which the dominant party is party 00 and the minimal winning coalitions are given by: $01-11-$ $10,00-01,00-11$, and $00-10$.

In this scenario, party 00 may decide to form a coalition with any of the other three parties, and each one of the other parties may decide to accept a proposal from party 00 or to join the three party coalition $01-11-10$. Thus there are four possible outcomes in this scenario. We find that coalition $00-01$ forms in equilibrium if and only if party 00's relative preference intensity for issue $x$ is large enough. Similarly, we can prove that coalition $00-10$ forms in equilibrium if and only if party 00's relative preference intensity for issue $x$ is small enough. We show that coalition 00-11 cannot be a part of an equilibrium. Otherwise, existence of equilibrium is only guaranteed under certain conditions and when they hold coalition $01-11-10$ forms. Finally, we show that coalition $00-11$ cannot be a part of an equilibrium.

\section{Theorem 2:}

In the "dominant party scenario" when $a_{00}=a_{11}$ and $a_{01}=a_{10}$ :

i) Coalition $00-01$ forms in equilibrium if and only if $a_{00} \geq \overline{a_{00}}$

ii) Coalition $00-10$ forms in equilibrium if and only if $a_{00} \leq \underline{a_{00}}$

iii) Coalition 01-11-10 forms in equilibrium if and only if $1 \leq \sqrt{a_{01}}\left(1-\sqrt{\frac{k_{00}}{a_{00}}}\right)+$ $\sqrt{b_{10}}\left(1-\sqrt{\frac{k_{00}}{b_{00}}}\right)$.

iv) Coalition $00-11$ never forms in equilibrium.

The two thresholds are given by: $\overline{a_{00}}=1-\left(\frac{1-\sqrt{k_{11}}}{1-\frac{1-\sqrt{k_{10}}}{\sqrt{b_{01}}}}\right)^{2}$ and $\underline{a_{00}}=\left(\frac{1-\sqrt{k_{11}}}{1-\frac{1-\sqrt{k_{01}}}{\sqrt{a_{10}}}}\right)^{2}$

This theorem characterizes the parameter values for which an equilibrium exists. Otherwise, we obtain cycles in the negotiations between the parties, in which case the governing coalitions that may form would be unstable. For $a_{00}=a_{11}$ and $a_{01}=a_{10}$ we have characterized the equilibrium outcomes for the dominant party scenario according to the values of $a_{00}$. We find that for values of $a_{00}$ small enough, the unique equilibrium coalition is $00-10$ and the set of equilibrium policies is given by

$$
A^{*}(00,10)=\left\{(x, 0) \in[0,1]^{2}: 1-\frac{1-\sqrt{k_{01}}}{\sqrt{a_{01}}} \leq x \leq \min \left\{\sqrt{\frac{b_{00}}{a_{00}}}\left(1-\sqrt{\frac{k_{01}}{b_{01}}}\right), \frac{1-\sqrt{k_{11}}}{\sqrt{a_{00}}}\right\}\right\}
$$

The range of values of $a_{00}$ for which coalition $00-10$ is part of an equilibrium outcome is larger if $k_{11}, k_{01}$, and $a_{10}$ are smaller (see figures 13 and 14).

Similarly, for values of $a_{00}$ large enough, the unique equilibrium coalition is $00-01$ and the set of equilibrium policies is given by

$$
A^{*}(00,01)=\left\{(0, y) \in[0,1]^{2}: 1-\frac{1-\sqrt{k_{10}}}{\sqrt{b_{01}}} \leq y \leq \min \left\{\sqrt{\frac{a_{00}}{b_{00}}}\left(1-\sqrt{\frac{k_{10}}{a_{10}}}\right), \frac{1-\sqrt{k_{11}}}{\sqrt{b_{00}}}\right\}\right\}
$$


The range of values of $a_{00}$ for which coalition $00-01$ is part of an equilibrium outcome is larger if $k_{11}, k_{10}$, and $b_{01}$ are smaller (see figures 15 and 16).

We have seen that coalition $00-11$ will never form in equilibrium (see figure 17) and that for intermediate values of $a_{00}$, coalition $01-11-10$ may form in equilibrium only if $a_{01}$ and $b_{10}$ are large enough and $k_{00}$ is small enough (see figure 18).

We have shown that the parameter value that determines which equilibrium prevails in this case is $a_{00}$ (see figure 19). Observe that the conditions for existence of equilibria with governing coalitions $00-01$ and $00-10$ are much weaker that those needed for the equilibria with governing coalition $01-11-$ 10. Indeed, the theorem states that it is most likely that the decision of the dominant party, party 00 in this case, will determine which governing coalition forms. Thus, the 'key party' in the 'dominant party scenario' coincides with the dominant party itself. Finally, observe that the intuition behind this result carries over to the case of $a_{00} \neq a_{11}$ and $a_{01} \neq a_{10}$, and the qualitative results obtained here can be reproduced in the more general case.

\section{Concluding remarks}

From the analysis of the model of government formation presented above we can draw the following implications:

i) coalitions of parties whose preferences differ on both issues are not likely

ii) three party coalitions are not likely

iii) it is sufficient that the key party has intense preferences over one the issues to guarantee the formation of a stable government coalition.

The main results, both about existence of equilibrium and about the composition of the equilibrium coalition, are determined by the value of the parties' relative intensity of preferences. This value represents the relative strength of a party's position over one of the issues and in the model we present this value is assumed to be exogenous. In reality this value is part of the party's private information, but given our results, a forward looking voter could use this value as a way to explain the party's decision and it could end up affecting the voting decision of policy motivated voters. In this the case, it might be in the party's interest to use the revelation of the value of its relative the intensity of preferences strategically. This observation points to an asymmetric information model of government formation.

Endogenous Value of Holding Office:

We have assumed that a party's value of holding office was exogenous. One could argue that a party's value of holding office today depends on its vote share, or on the proportion of parliament seats obtained by the party, if we were assuming proportional representation. Thus we could endogenize the present value of holding office by representing it as a function of the party's current vote share. Suppose that $k_{i}=k\left(v_{i}\right)$ with $k_{i}^{\prime}\left(v_{i}\right) \geq 0$. In this case we would be assuming that the larger the vote share of a party, the larger the utility that it derives from holding office, and the more it would be willing to compromise its 
policy position in order to become a member of the winning coalition. Thus, the sets of policies that are individually rational will be larger for parties with larger representation in the parliament.

This would imply that in the dominated party scenario, the coalition of parties that disagree on both issues would be less likely to form when the vote share of the key party is larger. Similarly, in the dominant party scenario, the three party coalition would be less likely to form when the vote share of the dominant party is larger.

Empty Sets of Acceptable Policies:

In the previous analysis we have assumed that the set of acceptable policies was not empty for all relevant coalition. If we assume that for some minimal winning coalitions the set of acceptable policies is empty. then the equilibrium conditions are relaxed: the analysis is simplified and existence of equilibrium exists for a wider range of parameter values.

Suppose that we are in the "dominated party scenario" and as before the dominated party is party 10 . If we assume that the set of acceptable policies by the winning coalition $00-11$ is empty, the only possible equilibrium coalitions are given by $00-01$, and $01-11$. In this case, party 01 has to decide which coalition to join: either $01-00$ or $01-11$. Party 01 will compare the outcomes it can obtain from each negotiation and decide to join the coalition whose final outcome gives it a larger utility. Observe that in this case existence of equilibrium is guaranteed for all parameter values and the larger is $a_{01}$ the more likely it is that party 01 ends up forming coalition $00-01$.

Now suppose that we are in the "dominant party scenario" and as before we assume that party 00 is the dominant party. If we assume that the set of acceptable policies by the three party coalition is empty, then party 00 has to decide which coalition to form: either $00-01,00-11$ or $00-10$. Notice that the larger is $a_{00}$ (the smaller is $b_{00}$ ) the more likely is that party 00 chooses to form coalition $00-01$; and the smaller is $a_{00}$ the more likely is that party 00 chooses to form coalition $00-10$.

Asymmetric Ideal Points:

An assumption that has simplified the calculus in this paper is the location of the ideal points of the four parties at the vertices of the unit square. This assumption could be relaxed considering, for instance $(0,1),(p, p),\left(p^{\prime}, p^{\prime}\right)$, and $(1,0)$, with $0<p<1 / 2<p$ ' $<1$.

As before, the relevant policy space will be delimited by the Pareto Set, which can be computed solving the corresponding maximization problems. For example, the following problem will give us as a solution the Pareto optimal policies of coalition $01-p p$ :

$\max -a_{01} x^{2}-b_{01}(1-y)^{2}$

s.t. $-a_{p p}(p-x)^{2}-b_{p p}(p-y)^{2} \geq u_{p p}$

From the first order conditions we obtain $-\frac{a_{01} x}{a_{p p}(x-p)}=\frac{b_{01}(1-y)}{b_{p p}(p-y)}$. This condition determines the set of Pareto optimal policies for coalition $01-p p$.

In particular, when $a_{01}=a_{p p}$ we have that $y=1-\frac{1-p}{p} x$ (see figure 20) and the contract curve is represented by a straight line. Otherwise, when $a_{01} \neq a_{p}$, 
we find that the contract curve would be concave or convex depending on the relative values of the preference intensity of the parties (see figure 21). Thus, it could be shown that all qualitative results of our basic model carry over to this more general case.

\section{References}

\section{References}

Austen-Smith, David and Jeffrey Banks (1988) "Elections, Coalitions and Legislative Outcomes" American Political Science Review 82: 405-22.

Axelrod, Robert (1970) Conflict of Interest, Chicago: Markham.

Baron, David and John Ferejohn (1989) "Bargaining in Legislatures" American Political Science Review 83: 1181-1207.

de Swaan, Abram (1973) Coalition Theories and Cabinet Formation. Amsterdam: Elsevier.

Diermeier, Daniel and Antonio Merlo (2004) "An Empirical Investigation of Coalitional Bargaining Procedures" Journal of Public Economics, 88, 783-797.

Diermeier, Daniel, Hulya Eraslan and Antonio Merlo (2003) "A Structural Model of Government Formation" Econometrica 71(1):27-70.

Enelow, James and Melvin Hinich (1983) "Voting One Issue at a Time: The Question of Voter Forecasts" American Political Science Review 27: 43545 .

Jackson, Matthew O. and Boaz Moselle (2002) "Coalition and Party Formation in a Legislative Voting Game" Journal of Economic Theory 103(1): 49-87.

Krehbiel, Keith (1988) "Spatial Models of Legislative Choice," Legislative Studies Quarterly 3: 259-319.

Laver, Michael and Norman Schofield (1990). Multiparty Government: The Politics of Coalition in Europe, Oxford University Press.

Leiserson, Michael (1966) Coalitions in Politics, Yale University, PhD Thesis.

McKelvey, Richard (1976) "Intransitivities in Multidimensional Voting Models and Some Implications for Agenda Control," Journal of Economic Theory 12 : 472-82.

McKelvey, Richard and Norman Schofield (1987) "Generalized Symmetry Conditions at a Core Point" Econometrica 55: 923-33.

Moulin, Herve (1988) Axioms of Cooperative Decision Making, Cambridge University Press.

Myerson, Roger (1991) Game Theory. Analysis of Conflict, Harvard University Press. 
Plott, Charles (1967) "A Notion of Equilibrium and its Possibility Under Majority Rule" American Economic Review 57: 787-806.

Riker, William (1962) The Theory of Political Coalitions, New Haven: Yale University Press.

Shepsle, Kenneth (1979) "Institutional Arrangements and Equilibrium in Multidimensional Voting Models" American Journal of Political Science 23: 27-60.

Von Neumann, John and Oskar Morgenstern (1953). Theory of Games and Economic Behavior, Princeton University Press.

\section{Appendix}

\section{Proof of Proposition 1:}

1) Since we have assumed that $v_{i}<\frac{1}{2}$ for all $i$, a coalition obtained by removing any party from any coalition of two parties may not be supported by a majority of the votes.

2) There are exactly six winning coalitions of two parties, and if a two party coalition is supported by a majority of the votes then its complementary is a two party coalition that cannot be a winning coalition.

3) Since we have assumed that $v_{i}<\frac{1}{2}$ for all $i$, any coalition formed by three parties is always supported by a majority of the votes.

4) Suppose that there is a minimal winning coalition that leaves party $i$ out. Then, all remaining coalitions of three parties must include party $i$ and all coalitions of two parties that include party $i$ are winning coalitions. Therefore, there cannot be any other minimal winning coalition of three parties.

\section{Proof of Proposition 2:}

i) Suppose that there is a minimal winning coalition that leaves party $i$ out. Then, all coalitions of two parties that include party $i$ are winning coalitions. There are exactly three coalitions of two parties that include party $i$. By proposition 1 there are exactly three winning coalitions of two parties. Therefore, party $i$ is a member of all minimal winning coalitions of two parties.

ii) Since there is no minimal winning coalition of three parties, from i) we know that no party can be a member of three winning coalitions of two parties. Since by proposition 1 there are exactly three winning coalitions of two parties, there must be at least one party included in exactly two winning coalitions of two parties. Suppose that it is party $i$ and we have that $v_{i}+v_{j}>\frac{1}{2}, v_{i}+v_{k}>\frac{1}{2}$, and $v_{i}+v_{l}<\frac{1}{2}$. This implies that $v_{k}+v_{l}<\frac{1}{2}, v_{j}+v_{l}<\frac{1}{2}$, and $v_{j}+v_{k}>\frac{1}{2}$. Thus party $l$ is not included in any of the winning coalitions. Therefore, there must be one party that is not included in any of the winning coalitions.

\section{Proof of Proposition 3:}

Consider coalition $00-01$. It is easy to see that Pareto optimal policies within the bargaining set of coalition $00-01$ must satisfy $(x, y)=(0, y)$ for any 
$y \in[0,1]$. Negotiating on the second dimension, party 00 will only accept policies that give it at least its reservation value, that is, values of $y$ such that $U_{00}(x, y)=$ $k_{00}-b_{00}(y)^{2} \geq 0$ or $y \leq \sqrt{\frac{k_{00}}{b_{00}}}$. Similarly, party 01 will only accept policies that give it at least its reservation value, that is, values of $y$ such that $U_{01}(x, y)=$ $k_{01}-b_{01}(1-y)^{2} \geq 0$ or $y \geq 1-\sqrt{\frac{k_{01}}{b_{01}}}$. Therefore, the set of policies supported by coalition $00-01$ is not empty if and only if $1 \leq \sqrt{\frac{k_{01}}{b_{01}}}+\sqrt{\frac{k_{00}}{b_{00}}}$. Notice that if $\frac{k_{i}}{b_{i}}>\frac{1}{2}$ for $i=00$ and 01 then this condition holds. In this case, there is a continuum of values for $y$ in the interval $[0,1]$ that are acceptable by both parties, given by the set: $A(00,01)=\left\{(0, y) \in[0,1]^{2}: 1-\sqrt{\frac{k_{01}}{b_{01}}} \leq y \leq \sqrt{\frac{k_{00}}{b_{00}}}\right\}$.

The ideal point of party 00 does not belong to this set if and only if $1-$ $\sqrt{\frac{k_{01}}{b_{01}}}>0$, that is $k_{01}<b_{01}$; and the ideal point of party 01 does not belong to this set if and only if $\sqrt{\frac{k_{00}}{b_{00}}}<1$, that is $k_{00}<b_{00}$.

The same argument could be applied to find the set of acceptable policies for coalition $11-10$.

\section{Proof of Proposition 4:}

Consider coalition $00-11$. In this case the Pareto optimal policies within the bargaining set of coalition $00-11$ are the solution to the following maximization problem:

$$
\begin{gathered}
\max k_{11}-a_{11}(1-x)^{2}-b_{11}(1-y)^{2} \\
\text { s.t. } k_{00}-a_{00}(x)^{2}-b_{00}(y)^{2} \geq 0
\end{gathered}
$$

From the first order conditions we obtain $\frac{a_{11}(1-x)}{b_{11}(1-y)}=\frac{a_{00} x}{b_{00} y}$. This condition determines the set of Pareto optimal policies for coalition $00-11$. Since we assume that $a_{00}=a_{11}$, we have that this set contains all $(x, y)$ such that $x=$ $y \in(0,1)$.

The subset of Pareto optimal policies that are accepted by coalition $00-11$ has to provide each party in the coalition with a utility level of at least its reservation value, that is, they have to satisfy the following conditions: $U_{00}(x, x)=$ $k_{00}-a_{00} x^{2}-b_{00} x^{2} \geq 0$ and $U_{11}(x, x)=k_{11}-a_{00}(1-x)^{2}-b_{00}(1-x)^{2} \geq 0$. Thus we must have that $x \leq \sqrt{k_{00}}$ and $x \geq 1-\sqrt{k_{11}}$. Observe that there is a value of $x$ such that $1-\sqrt{k_{11}} \leq x \leq \sqrt{k_{00}}$ if and only if $1 \leq \sqrt{k_{11}}+\sqrt{k_{00}}$.

Therefore, the set of policies that are acceptable by coalition $00-11$ when $a_{00}=a_{11}$ is $A\left(00,11 ; a_{00}=a_{11}\right)=\left\{(x, x) \in[0,1]^{2}: 1-\sqrt{k_{11}} \leq x \leq \sqrt{k_{00}}\right\}$.

The ideal point of party 00 does not belong to this set of policies acceptable by this coalition if and only if $U_{11}(0,0)=k_{11}-a_{11}-b_{11}=-1<0$, that is, $k_{11}<1$. Similarly, the ideal point of party 11 does not belong to this set if and only if $U_{00}(1,1)=k_{00}-a_{00}-b_{00}=-1<0$, that is, $k_{00}<1$.

A similar argument can be applied to characterize the set of acceptable policies by coalition $01-10$. 


\section{Proof of Proposition 5:}

A sufficient condition for non emptiness of the set of Individually Rational policies for parties 00 and 11 is the following: $U_{i}\left(\frac{1}{2}, y\right)=0$ for $y>\frac{1}{2}$, for $i \in\{00,11\}$ and for all $a_{i} \in(0,1)$. (See figure 9)

Consider the case of party 00 . We have that $U_{00}\left(\frac{1}{2}, y\right)=k_{00}-a_{00}\left(\frac{1}{2}\right)^{2}-$ $b_{00}(y)^{2}=0$. Therefore, $y=\sqrt{\frac{k_{00}-\frac{a_{00}}{4}}{b_{00}}}>\frac{1}{2}$ if and only if $k_{00}-\frac{a_{00}}{4}>\frac{1-a_{00}}{4}$ if and only if $k_{00}>\frac{1}{4}$.

Since this result holds for all values of $a_{00} \in(0,1)$, then with a symmetric argument we also obtain that if $U_{11}\left(\frac{1}{2}, y\right)=0$ then $y<\frac{1}{2}$ for all values of $a_{11} \in(0,1)$ when $k_{11}>\frac{1}{4}$.

Thus we have shown that if $k_{i}>\frac{1}{4}$ for all $i$, generically there is a continuum of policies in the set of acceptable policies by coalition $00-11$. (See figure 10)

The ideal point of party 00 does not belong to this set if and only if $U_{11}(0,0)=$ $k_{11}-a_{11}-b_{11}=k_{11}-1<0$, that is, $k_{11}<1$. Similarly, the ideal point of party 11 does not belong to this set if and only if $U_{00}(1,1)=k_{00}-a_{00}-b_{00}=k_{00}-1<0$, that is, $k_{00}<1$.

A similar argument can be applied to characterize the set of acceptable policies by coalition $01-10$.

\section{Proof of Theorem 1:}

Since there are three minimal winning coalitions, there are three possible equilibrium coalitions in this scenario: $00-01,01-11$, and $00-11$.

1) Coalition $00-01$ : In this case the policy outcome will be a policy in the set of policies supported by coalition $00-01$ that gives party 00 and party 01 at least the same utility that they could obtain from the best offer they could have from party 11 .

In order to have coalition $00-01$ forming we need to show that there is a policy supported by this coalition that gives party 00 and party 01 at least the same utility that they could obtain from the best offer they could have from party 11. First notice that since $U_{11}\left(1-\sqrt{\frac{k_{11}}{a_{11}}}, 1\right)=0$, the best offer that party 01 may obtain from party 11 gives it a utility level equal to: $U_{01}\left(1-\sqrt{\frac{k_{11}}{a_{11}}}, 1\right)=$ $k_{01}-a_{01}\left(1-\sqrt{\frac{k_{11}}{a_{11}}}\right)^{2}$. Notice that $U_{01}\left(1-\sqrt{\frac{k_{11}}{a_{11}}}, 1\right)>0$ from proposition 3. The possible offers from party 00 that give party 01 a utility level of at least $k_{01}-a_{01}\left(1-\sqrt{\frac{k_{11}}{a_{11}}}\right)^{2}$ have to satisfy that $U_{01}(0, y)=k_{01}-b_{01}(1-y)^{2} \geq$ $k_{01}-a_{01}\left(1-\sqrt{\frac{k_{11}}{a_{11}}}\right)^{2}$, that is, $y \geq 1-\sqrt{\frac{a_{01}}{b_{01}}}\left(1-\sqrt{\frac{k_{11}}{a_{11}}}\right)$.

Next observe that if $a_{00}=a_{11}$, the best offer that party 00 may obtain from party 11 gives it a utility level equal to: $U_{00}\left(1-\sqrt{k_{11}}, 1-\sqrt{k_{11}}\right)=$ $k_{00}-\left(1-\sqrt{k_{11}}\right)^{2}$. Notice that $U_{00}\left(1-\sqrt{k_{11}}, 1-\sqrt{k_{11}}\right)>k_{00}-\left(1-v_{00}\right)$ from proposition 5 . The possible offers from party 01 that give party 00 a utility level of $k_{00}-\left(1-\sqrt{k_{11}}\right)^{2}$ have to satisfy that $U_{00}(0, y)=k_{00}-b_{00}(y)^{2} \geq$ $k_{00}-\left(1-\sqrt{k_{11}}\right)^{2}$, that is, $y \leq \frac{1-\sqrt{k_{11}}}{\sqrt{b_{00}}}$. 
Thus the policy that is supported by coalition $00-01$ and gives party 00 and party 01 at least the same utility that they could obtain from the best offer they could have from party 11, has to satisfy:

$1-\sqrt{\frac{a_{01}}{b_{01}}}\left(1-\sqrt{\frac{k_{11}}{a_{11}}}\right) \leq y \leq \frac{1-\sqrt{k_{11}}}{\sqrt{b_{00}}}$.

Such a policy exists if and only if $1-\sqrt{\frac{a_{01}}{b_{01}}}\left(1-\sqrt{\frac{k_{11}}{a_{11}}}\right) \leq \frac{1-\sqrt{k_{11}}}{\sqrt{b_{00}}}$, which can be written as $\left.a_{01} \geq \frac{1}{\left(\frac{1-\sqrt{\frac{k_{11}}{a_{11}}}}{2}\right.}\right)^{2}$. Thus, we need $a_{01}$ to be large enough. In $1+\left(\frac{1-\sqrt{\frac{k_{11}}{a_{11}}}}{1-\frac{1-\sqrt{k_{11}}}{\sqrt{b_{00}}}}\right)$

this case, coalition $00-01$ forms and the policy outcome is the set $A^{*}(00-01)=$ $\left\{(0, y) \in[0,1]^{2}: 1-\sqrt{\frac{a_{01}}{b_{01}}}\left(1-\sqrt{\frac{k_{11}}{a_{11}}}\right) \leq y \leq \frac{1-\sqrt{k_{11}}}{\sqrt{b_{00}}}\right\}$. See figure 10 .

2) Coalition $01-11$ : In this case the policy outcome will be a policy in the set of policies supported by coalition $01-11$ that gives party 01 and party 11 at least the same utility that they could obtain from the best offer they could have from party 00 .

In order to have coalition $01-11$ forming we need to show that there is a policy supported by this coalition that gives party 01 and party 11 at least the same utility that they could obtain from the best offer they could have from party 00 . First notice that since $U_{00}\left(0, \sqrt{\frac{k_{00}}{b_{00}}}\right)=0$, the best offer that party 01 may obtain from party 00 gives it a utility level equal to: $U_{01}\left(0, \sqrt{\frac{k_{00}}{b_{00}}}\right)=k_{01}-b_{01}\left(1-\sqrt{\frac{k_{00}}{b_{00}}}\right)^{2}$. Notice that $U_{01}\left(0, \sqrt{\frac{k_{00}}{b_{00}}}\right)>0$ from proposition 4 . The possible offers from party 11 that give party 01 a utility level of $k_{01}-b_{01}\left(1-\sqrt{\frac{k_{00}}{b_{00}}}\right)^{2}$ have to satisfy that $U_{01}(x, 1)=k_{01}-a_{01} x^{2} \geq$ $k_{01}-b_{01}\left(1-\sqrt{\frac{k_{00}}{b_{00}}}\right)^{2}$, that is, $x \leq \sqrt{\frac{b_{01}}{a_{01}}}\left(1-\sqrt{\frac{k_{00}}{b_{00}}}\right)$.

Next observe that if $a_{00}=a_{11}$, the best offer that party 11 may obtain from party 00 gives it a utility level equal to: $U_{11}\left(\sqrt{k_{00}}, \sqrt{k_{00}}\right)=k_{11}-\left(1-\sqrt{k_{00}}\right)^{2}$. Notice that $U_{11}\left(\sqrt{k_{00}}, \sqrt{k_{00}}\right)>0$ from proposition 5 . The possible offers from party 01 that give party 11 a utility level of $k_{11}-\left(1-\sqrt{k_{00}}\right)^{2}$ have to satisfy that $U_{11}(x, 1)=k_{11}-a_{11}(1-x)^{2} \geq k_{11}-\left(1-\sqrt{k_{00}}\right)^{2}$, that is, $x \geq 1-\frac{1-\sqrt{k_{00}}}{\sqrt{a_{11}}}$.

Thus the policy that is supported by coalition $01-11$ and gives party 01 and party 11 at least the same utility that they could obtain from the best offer they could have from party 00, has to satisfy:

$$
1-\frac{1-\sqrt{k_{00}}}{\sqrt{a_{11}}} \leq x \leq \sqrt{\frac{b_{01}}{a_{01}}}\left(1-\sqrt{\frac{k_{00}}{b_{00}}}\right)
$$

Such a policy exists if and only if $1-\frac{1-\sqrt{k_{00}}}{\sqrt{a_{11}}} \leq \sqrt{\frac{b_{01}}{a_{01}}}\left(1-\sqrt{\frac{k_{00}}{b_{00}}}\right)$, which can be written as $a_{01} \leq \frac{1}{1+\left(\frac{1-\frac{1-\sqrt{k_{00}}}{\sqrt{a_{11}}}}{1-\sqrt{\frac{k_{00}}{b_{00}}}}\right)^{2}}$. Thus, we need $a_{01}$ to be small enough. In this case, coalition $01-11$ forms and the policy outcome is the set $A^{*}(01-11)=$ $\left\{(x, 1) \in[0,1]^{2}: 1-\frac{1-\sqrt{k_{00}}}{\sqrt{a_{11}}} \leq x \leq \sqrt{\frac{b_{01}}{a_{01}}}\left(1-\sqrt{\frac{k_{00}}{b_{00}}}\right)\right\}$. 
See figure 9 .

3) Coalition $00-11$ : In this case the policy outcome will be a policy in the set of policies supported by coalition $00-11$ that gives party 00 and party 11 at least the same utility that they could obtain from the best offer they could have from party 01 .

In order to have coalition $00-11$ forming we need to show that there is a policy supported by this coalition that gives party 00 and party 11 at least the same utility that they could obtain from the best offer they could have from party 01.

First notice that the best offer that party 00 can obtain from party 01 is $\left(0,1-\sqrt{\frac{k_{01}}{b_{01}}}\right)$, and the best offer that party 11 can obtain from party 01 is $\left(\sqrt{\frac{k_{01}}{a_{01}}}, 1\right)$.

We need to show that there is a policy $(x, y)$ such that it is acceptable by coalition $00-11,(x, y) \in A(00-11)$ and such that $U_{00}(x, y) \geq U_{00}\left(0,1-\sqrt{\frac{k_{01}}{b_{01}}}\right)=$ $k_{00}-b_{00}\left(1-\sqrt{\frac{k_{01}}{b_{01}}}\right)^{2}$ and $U_{11}(x, y) \geq U_{11}\left(\sqrt{\frac{k_{01}}{a_{01}}}, 1\right)=k_{11}-a_{11}\left(1-\sqrt{\frac{k_{01}}{a_{01}}}\right)^{2}$.

If we assume that $a_{00}=a_{11}$ we have that $(x, y)=(x, x)$, thus we need to show that there is a value of $x \in[0,1]$ such that $k_{00}-x^{2} \geq k_{00}-b_{00}\left(1-\sqrt{\frac{k_{01}}{b_{01}}}\right)^{2}$ and $k_{11}-(1-x)^{2} \geq k_{11}-a_{11}\left(1-\sqrt{\frac{k_{01}}{a_{01}}}\right)^{2}$

therefore, we need $x \leq \sqrt{b_{00}}\left(1-\sqrt{\frac{k_{01}}{b_{01}}}\right)$ and $x \geq 1-\sqrt{a_{11}}\left(1-\sqrt{\frac{k_{01}}{a_{01}}}\right)$

Thus, the equilibrium policies for coalition $00-11$ will be those $(x, x) \in$ $A(00-11)$ such that $1-\sqrt{a_{11}}\left(1-\sqrt{\frac{k_{01}}{a_{01}}}\right) \leq x \leq \sqrt{b_{00}}\left(1-\sqrt{\frac{k_{01}}{b_{01}}}\right)$.

Since we assume that the sets of acceptable policies by all wining coalitions are not empty, by propositions 3 and 4 we have that $1-\sqrt{k_{11}} \leq 1-$ $\sqrt{a_{11}}\left(1-\sqrt{\frac{k_{01}}{a_{01}}}\right)$ and $\sqrt{b_{00}}\left(1-\sqrt{\frac{k_{01}}{b_{01}}}\right) \leq \sqrt{k_{00}}$.

Since, we have that the set of acceptable policies by coalition $00-11$ is

$A\left(00-11 ; a_{00}=a_{11}\right)=\left\{(x, x) \in[0,1]^{2}: 1-\sqrt{k_{11}} \leq x \leq \sqrt{k_{00}}\right\}$

We conclude a necessary and sufficient condition for this equilibrium to exist is

$1 \leq \sqrt{a_{11}}\left(1-\sqrt{\frac{k_{01}}{a_{01}}}\right)+\sqrt{b_{00}}\left(1-\sqrt{\frac{k_{01}}{b_{01}}}\right)$.

This condition is more likely to be satisfied the larger $a_{11}$ and $b_{00}$ are and the smaller $k_{01}$ is. See figure 11 .

Proof of Theorem 2:

Since there are four minimal winning coalitions, there are four possible equilibrium coalitions in this scenario; $00-01,00-11,00-10$, and $01-11-10$.

1) Coalition $00-01$ : In this case the policy outcome will be a policy in the set of policies supported by coalition $00-01$ that: gives party 00 at least the same utility that it could obtain from joining either party 11 or party 10 in a coalition, and gives party 01 least the same utility that it could obtain from joining the three party coalition. 
Formally, coalition $00-01$ can be part of an equilibrium outcome if and only if there is a value $\widetilde{y} \in[0,1]$ such that:

i) $U_{01}(0, \widetilde{y}) \geq U_{01}\left(x_{01}(01-11-10), y_{01}(01-11-10)\right)$

ii) $U_{00}(x, y)<U_{00}(0, \widetilde{y})$ for all $(x, y)$ such that $U_{10}(x, y) \geq 0$

iii) $U_{00}(x, y)<U_{00}(0, \widetilde{y})$ for all $(x, y)$ such that $U_{11}(x, y) \geq 0$

iv) $U_{00}(0, \widetilde{y}) \geq 0$

Condition (i) implies that $\widetilde{y}$ has to satisfy: $U_{01}(0, \widetilde{y})=k_{01}-b_{01}(1-\widetilde{y})^{2} \geq$ $k_{01}-\left(1-\sqrt{k_{10}}\right)^{2}=U_{01}\left(x_{01}(01-11-10), y_{01}(01-11-10)\right)$, that is, $\widetilde{y} \geq$ $1-\frac{1-\sqrt{k_{10}}}{\sqrt{b_{01}}}$.

To prove condition (ii) it suffices show that for $x$ such that $U_{10}(x, 0)=k_{10}-$ $a_{10}(1-x)^{2}=0$, that is, $x=1-\sqrt{\frac{k_{10}}{a_{10}}}$, we must have that $U_{00}\left(1-\sqrt{\frac{k_{10}}{a_{10}}}, 0\right)=$ $k_{00}-a_{00}\left(1-\sqrt{\frac{k_{10}}{a_{10}}}\right)^{2} \leq k_{00}-b_{00}(\widetilde{y})^{2}=U_{00}(0, \widetilde{y})$, that is, $\widetilde{y} \leq \sqrt{\frac{a_{00}}{b_{00}}}\left(1-\sqrt{\frac{k_{10}}{a_{10}}}\right)$.

To prove condition (iii) it suffices to show that for $x$ such that $U_{11}(x, x)=$ $k_{11}-(1-x)^{2}=0$, that is, $x=1-\sqrt{k_{11}}$, we must have that $U_{00}\left(1-\sqrt{k_{11}}, 1-\sqrt{k_{11}}\right)=$ $k_{00}-\left(1-\sqrt{k_{11}}\right)^{2}<k_{00}-b_{00}(\widetilde{y})^{2}=U_{00}(0, \widetilde{y})$, that is, $\widetilde{y} \leq \frac{1-\sqrt{k_{11}}}{\sqrt{b_{00}}}$.

Condition (iv) is always satisfied since we assume that the sets of acceptable policies for each coalition are not empty.

Thus, this coalition will form in equilibrium if and only if:

$1 \leq \frac{1-\sqrt{k_{10}}}{\sqrt{b_{01}}}+\min \left\{\sqrt{\frac{a_{00}}{b_{00}}}\left(1-\sqrt{\frac{k_{10}}{a_{10}}}\right), \frac{1-\sqrt{k_{11}}}{\sqrt{b_{00}}}\right\}$

And the policy implemented in this case is $(0, \widetilde{y})$ such that

$1-\frac{1-\sqrt{k_{10}}}{\sqrt{b_{01}}} \leq \widetilde{y} \leq \min \left\{\sqrt{\frac{a_{00}}{b_{00}}}\left(1-\sqrt{\frac{k_{10}}{a_{10}}}\right), \frac{1-\sqrt{k_{11}}}{\sqrt{b_{00}}}\right\}$

Observe that $\sqrt{\frac{a_{00}}{b_{00}}}\left(1-\sqrt{\frac{k_{10}}{a_{10}}}\right) \leq \frac{1-\sqrt{k_{11}}}{\sqrt{b_{00}}}$ if and only if $a_{00} \leq\left(\frac{1-\sqrt{k_{11}}}{1-\sqrt{\frac{k_{10}}{a_{10}}}}\right)^{2}$.

In this case the condition that has to be satisfied is $1 \leq \frac{1-\sqrt{k_{10}}}{\sqrt{b_{01}}}+\sqrt{\frac{a_{00}}{b_{00}}}\left(1-\sqrt{\frac{k_{10}}{a_{10}}}\right)$.

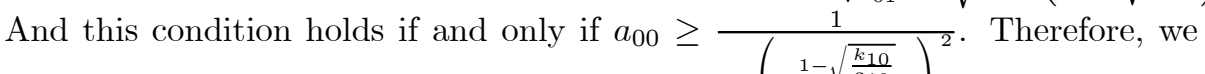

$$
1+\left(\frac{1-\sqrt{\frac{k_{10}}{a_{10}}}}{1-\frac{1-\sqrt{k_{10}}}{\sqrt{b_{01}}}}\right)
$$

need $\frac{1}{1+\left(\frac{1-\sqrt{\frac{k_{10}}{a_{10}}}}{1-\frac{1-\sqrt{k_{10}}}{\sqrt{b_{01}}}}\right)^{2}} \leq a_{00} \leq\left(\frac{1-\sqrt{k_{11}}}{1-\sqrt{\frac{k_{10}}{a_{10}}}}\right)^{2}$

Such a value for $a_{00}$ exists if and only if $\frac{1}{1+\left(\frac{1-\sqrt{\frac{k_{10}}{a_{10}}}}{1-\frac{1-\sqrt{k_{10}}}{\sqrt{b_{01}}}}\right)^{2}} \leq\left(\frac{1-\sqrt{k_{11}}}{1-\sqrt{\frac{k_{10}}{a_{10}}}}\right)^{2}$.

Otherwise, we have that $\sqrt{\frac{a_{00}}{b_{00}}}\left(1-\sqrt{\frac{k_{10}}{a_{10}}}\right) \geq \frac{1-\sqrt{k_{11}}}{\sqrt{b_{00}}}$ if and only if $a_{00} \geq$ $\left(\frac{1-\sqrt{\frac{k_{1}}{k_{10}}}}{1-\sqrt{\frac{k_{10}}{a_{10}}}}\right)^{2}$. In this case the condition that has to be satisfied is $1 \leq \frac{1-\sqrt{k_{10}}}{\sqrt{b_{01}}}+$ 
$\frac{1-\sqrt{k_{11}}}{\sqrt{b_{00}}}$. And this condition holds if and only if $a_{00} \geq 1-\left(\frac{1-\sqrt{k_{11}}}{1-\frac{1-\sqrt{k_{10}}}{\sqrt{b_{01}}}}\right)^{2}$. Therefore, we need $a_{00} \geq \max \left\{\left(\frac{1-\sqrt{k_{11}}}{1-\sqrt{\frac{k_{10}}{a_{10}}}}\right)^{2}, 1-\left(\frac{1-\sqrt{k_{11}}}{1-\frac{1-\sqrt{k_{10}}}{\sqrt{b_{01}}}}\right)^{2}\right\}$.

It can be shown that $\left(\frac{1-\sqrt{k_{11}}}{1-\sqrt{\frac{k_{10}}{a_{10}}}}\right)^{2} \geq 1-\left(\frac{1-\sqrt{k_{11}}}{1-\frac{1-\sqrt{k_{10}}}{\sqrt{b_{01}}}}\right)^{2}$ if and only if $\frac{1}{1+\left(\frac{1-\sqrt{\frac{k_{10}}{a_{10}}}}{1-\frac{1-\sqrt{k_{10}}}{\sqrt{b_{01}}}}\right)^{2}} \leq\left(\frac{1-\sqrt{k_{11}}}{1-\sqrt{\frac{k_{10}}{a_{10}}}}\right)^{2}$

In particular we have coalition 00-01 in equilibrium for all $a_{00} \geq$

$1-\left(\frac{1-\sqrt{k_{11}}}{1-\frac{1-\sqrt{k_{10}}}{\sqrt{b_{01}}}}\right)^{2}$ and:

$\frac{1}{1+\left(\frac{1-\sqrt{\frac{k_{10}}{a_{10}}}}{1-\frac{1-\sqrt{k_{10}}}{\sqrt{b_{01}}}}\right)^{2}}=$

i) If $\frac{1}{1+\left(\frac{1-\sqrt{\frac{k_{10}}{a_{10}}}}{1-\frac{1-\sqrt{k_{10}}}{\sqrt{b_{01}}}}\right)^{2}} \leq\left(\frac{1-\sqrt{k_{11}}}{1-\sqrt{\frac{k_{10}}{a_{10}}}}\right)^{2}$ the equilibrium conditions are given by the potential offers to party 00 from parties 10 and 11 . See figure 15 .

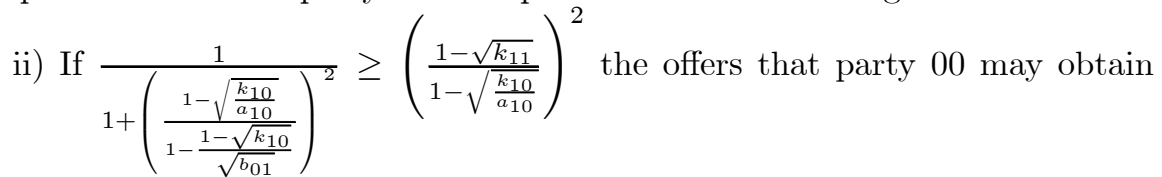

from party 10 are not binding, thus the conditions are given by the potential offers from party 11 . See figure 16 .

2) Coalition $00-10$ : In this case the policy outcome will be a policy in the set of policies supported by coalition $00-10$ that: gives party 00 at least the same utility that it could obtain from joining either party 01 or party 11 in a coalition, and gives party 10 least the same utility that it could obtain from joining the three party coalition.

Formally, coalition $00-10$ can be part of an equilibrium outcome if and only if there is a value $\widetilde{x} \in[0,1]$ such that:

i) $U_{10}(\widetilde{x}, 0) \geq U_{10}\left(x_{10}(01-11-10), y_{10}(01-11-10)\right)$

ii) $U_{00}(x, y)<U_{00}(\widetilde{x}, 0)$ for all $(x, y)$ such that $U_{01}(x, y) \geq 0$

iii) $U_{00}(x, y)<U_{00}(\widetilde{x}, 0)$ for all $(x, y)$ such that $U_{11}(x, y) \geq 0$

iv) $U_{00}(\widetilde{x}, 0) \geq 0$

See figures 13 and 14 . Condition (i) implies that $\widetilde{x}$ has to satisfy: $U_{10}(\widetilde{x}, 0)=$ $k_{10}-a_{10}(1-\widetilde{x})^{2} \geq k_{10}-\left(1-\sqrt{k_{01}}\right)^{2}=U_{10}\left(x_{10}(01-11-10), y_{10}(01-11-10)\right)$, that is, $\widetilde{x} \geq 1-\frac{1-\sqrt{k_{01}}}{\sqrt{a_{10}}}$.

To prove condition (ii) it suffices show that for $y$ such that $U_{01}(0, y)=k_{01}-$ 
$b_{01}(1-y)^{2}=0$, that is, $y=1-\sqrt{\frac{k_{01}}{b_{01}}}$ we must have that $U_{00}\left(0,1-\sqrt{\frac{k_{01}}{b_{01}}}\right)=$ $k_{00}-b_{00}\left(1-\sqrt{\frac{k_{01}}{b_{01}}}\right)^{2} \leq k_{00}-a_{00}(\widetilde{x})^{2}=U_{00}(\widetilde{x}, 0)$, that is, $\widetilde{x} \leq \sqrt{\frac{b_{00}}{a_{00}}}\left(1-\sqrt{\frac{k_{01}}{b_{01}}}\right)$.

To prove condition (iii) it suffices to show that for $x$ such that $U_{11}(x, x)=$ $k_{11}-(1-x)^{2}=0$, that is, $x=1-\sqrt{k_{11}}$, we must have that $U_{00}\left(1-\sqrt{k_{11}}, 1-\sqrt{k_{11}}\right)=$ $k_{00}-\left(1-\sqrt{k_{11}}\right)^{2}<k_{00}-a_{00}(\widetilde{x})^{2}=U_{00}(0, \widetilde{x})$, that is, $\widetilde{x} \leq \frac{1-\sqrt{k_{11}}}{\sqrt{a_{00}}}$.

Condition (iv) is always satisfied since we assume that all sets of acceptable policies are not empty.

Thus, this coalition will form in equilibrium if and only if:

$1 \leq \frac{1-\sqrt{k_{01}}}{\sqrt{a_{01}}}+\min \left\{\sqrt{\frac{b_{00}}{a_{00}}}\left(1-\sqrt{\frac{k_{01}}{b_{01}}}\right), \frac{1-\sqrt{k_{11}}}{\sqrt{a_{00}}}\right\}$

And the policy implemented in this case is $(\widetilde{x}, 0)$ such that

$1-\frac{1-\sqrt{k_{01}}}{\sqrt{a_{01}}} \leq \widetilde{x} \leq \min \left\{\sqrt{\frac{b_{00}}{a_{00}}}\left(1-\sqrt{\frac{k_{01}}{b_{01}}}\right), \frac{1-\sqrt{k_{11}}}{\sqrt{a_{00}}}\right\}$

Observe that $\sqrt{\frac{b_{00}}{a_{00}}}\left(1-\sqrt{\frac{k_{01}}{b_{01}}}\right) \leq \frac{1-\sqrt{k_{11}}}{\sqrt{a_{00}}}$ if and only if $b_{00} \leq\left(\frac{1-\sqrt{k_{11}}}{1-\sqrt{\frac{k_{01}}{b_{01}}}}\right)^{2}$.

In this case the condition that has to be satisfied is $1 \leq \frac{1-\sqrt{k_{01}}}{\sqrt{a_{10}}}+\sqrt{\frac{b_{00}}{a_{00}}}\left(1-\sqrt{\frac{k_{01}}{b_{01}}}\right)$.

And this condition holds if and only if $\left.b_{00} \geq \frac{1}{1+\left(\frac{1-\sqrt{k_{01}}}{1-\frac{1-\sqrt{k_{01}}}{\sqrt{v_{10}}}}\right)}\right)^{2}$. Therefore, we need $\frac{1}{1+\left(\frac{1-\sqrt{\frac{k_{01}}{b_{01}}}}{1-\frac{1-\sqrt{k_{01}}}{\sqrt{a_{10}}}}\right)^{2}} \leq b_{00} \leq\left(\frac{1-\sqrt{k_{11}}}{1-\sqrt{\frac{k_{01}}{b_{01}}}}\right)^{2}$.Such a value for $b_{00}$ exists if and only if $\frac{1}{1+\left(\frac{1-\sqrt{\frac{k_{01}}{b_{01}}}}{1-\frac{1-\sqrt{k_{01}}}{\sqrt{a_{10}}}}\right)^{2}} \leq\left(\frac{1-\sqrt{k_{11}}}{1-\sqrt{\frac{k_{k_{01}}}{b_{01}}}}\right)^{2}$.

Otherwise, $\sqrt{\frac{b_{00}}{a_{00}}}\left(1-\sqrt{\frac{k_{01}}{b_{01}}}\right) \geq \frac{1-\sqrt{k_{11}}}{\sqrt{a_{00}}}$ if and only if $b_{00} \geq\left(\frac{1-\sqrt{k_{11}}}{1-\sqrt{\frac{k_{01}}{b_{01}}}}\right)^{2}$. In this case the condition that has to be satisfied is $1 \leq \frac{1-\sqrt{k_{01}}}{\sqrt{a_{10}}}+\frac{1-\sqrt{k_{11}}}{\sqrt{a_{00}}}$. And this condition holds if and only if $b_{00} \geq 1-\left(\frac{1-\sqrt{k_{11}}}{1-\frac{1-\sqrt{k_{01}}}{\sqrt{a_{10}}}}\right)^{2}$. Therefore, we need $b_{00} \geq \max \left\{\left(\frac{1-\sqrt{k_{11}}}{1-\sqrt{\frac{k_{01}}{b_{01}}}}\right)^{2}, 1-\left(\frac{1-\sqrt{k_{11}}}{1-\frac{1-\sqrt{k_{01}}}{\sqrt{a_{10}}}}\right)^{2}\right\}$.

It can be shown that $\left(\frac{1-\sqrt{k_{11}}}{1-\sqrt{\frac{k_{01}}{b_{01}}}}\right)^{2} \geq 1-\left(\frac{1-\sqrt{k_{11}}}{1-\frac{1-\sqrt{k_{10}}}{\sqrt{a_{10}}}}\right)^{2}$ if and only if $\frac{1}{1+\left(\frac{1-\sqrt{\frac{k_{01}}{b_{0}}}}{1-\frac{1-\sqrt{k_{01}}}{\sqrt{\overline{1}_{10}}}}\right)^{2}} \leq$ 


$$
\left(\frac{1-\sqrt{k_{11}}}{1-\sqrt{\frac{k_{01}}{b_{01}}}}\right)^{2}
$$

Therefore, we must have $1-\left(\frac{1-\sqrt{k_{11}}}{1-\frac{1-\sqrt{k_{01}}}{\sqrt{\bar{a}_{10}}}}\right)^{2}=\frac{1}{1+\left(\frac{1-\sqrt{k_{01}}}{1-\frac{1-\sqrt{k_{01}}}{\sqrt{a_{10}}}}\right)^{2}}$.

In particular we have coalition $00-10$ in equilibrium for all $b_{00} \geq$

and:

$$
\frac{1}{1+\left(\frac{1-\sqrt{\frac{k_{01}}{b_{01}}}}{1-\frac{1-\sqrt{k_{01}}}{\sqrt{a_{10}}}}\right)^{2}}
$$

i) If $\frac{1}{1+\left(\frac{1-\sqrt{\frac{k_{01}}{b_{01}}}}{1-\frac{1-\sqrt{k_{01}}}{\sqrt{\bar{k}_{10}}}}\right)^{2}} \leq\left(\frac{1-\sqrt{k_{11}}}{1-\sqrt{\frac{k_{0_{01}}}{b_{01}}}}\right)^{2}$ the equilibrium conditions are given by the potential offers to party 00 from parties 01 and 11 .

ii) If $\frac{1}{1+\left(\frac{1-\sqrt{\frac{k_{01}}{b_{01}}}}{1-\frac{1-\sqrt{k_{01}}}{\sqrt{a_{10}}}}\right)^{2}} \geq\left(\frac{1-\sqrt{k_{11}}}{1-\sqrt{\frac{k_{01}}{b_{01}}}}\right)^{2}$ the offers that party 00 may obtain from party 01 are not binding, thus the conditions are given by the potential offers from party 11.

3) Coalition $01-11-10$ : In this case the policy outcome will be a policy in the set of policies supported by the three party coalition $01-11-10$ that gives party 01 , party 11 , and party 10 at least the same utility that they could obtain from the best offer they could have from party 00 .

In order to have coalition $01-11-10$ forming we need to show that there is a policy in the set of acceptable policies by the three party coalition such that each party in the coalition prefers this policy to the best offer that party 00 could make. Since we have already shown that there is no offer from party 00 to party 11 that would be preferred by the later to a policy that is acceptable by the coalition of three parties, we only need to take care of offers from party 00 to parties 01 and 10 .

Thus, it is necessary that the best offer from party 00 to parties 01 and 10 is not preferred by these parties to best offer that they can obtain from coalition $01-11-10$. First notice that the best offer that party 01 can obtain from party 00 is $\left(0, \sqrt{\frac{k_{00}}{b_{00}}}\right)$, and the best offer that party 10 can obtain from party 00 is $\left(\sqrt{\frac{k_{00}}{a_{00}}}, 0\right)$.

We will first show that there is a policy $(x, y)$ such that it is acceptable by coalition 01-10, $(x, y) \in A(01-10)$ and such that $U_{01}(x, y) \geq U_{01}\left(0, \sqrt{\frac{k_{00}}{b_{00}}}\right)=$ $k_{00}-b_{01}\left(\sqrt{\frac{k_{00}}{b_{00}}}\right)^{2}$ and $U_{10}(x, y) \geq U_{10}\left(\sqrt{\frac{k_{00}}{a_{00}}}, 0\right)=k_{11}-a_{10}\left(1-\sqrt{\frac{k_{00}}{a_{00}}}\right)^{2}$.

If we assume that $a_{01}=a_{10}$ we have that $(x, y)=(x, 1-x)$, thus we need to show that there is a value of $x \in[0,1]$ such that $k_{01}-x^{2} \geq k_{00}-b_{01}\left(\sqrt{\frac{k_{00}}{b_{00}}}\right)^{2}$ 
and $k_{11}-(1-x)^{2} \geq k_{11}-a_{10}\left(1-\sqrt{\frac{k_{00}}{a_{00}}}\right)^{2}$

therefore, we need $x \leq \sqrt{b_{01}}\left(1-\sqrt{\frac{k_{00}}{b_{00}}}\right)$ and $x \geq 1-\sqrt{a_{10}}\left(1-\sqrt{\frac{k_{00}}{a_{00}}}\right)$

Thus, the equilibrium policies for coalition $01-10$ will be those $(x, x) \in$ $A(01-10)$ such that $1-\sqrt{a_{10}}\left(1-\sqrt{\frac{k_{00}}{a_{00}}}\right) \leq x \leq \sqrt{b_{01}}\left(1-\sqrt{\frac{k_{00}}{b_{00}}}\right)$.

Since, we assume that the sets of acceptable policies by all relevant coalitions are not empty, we have that a necessary and sufficient condition for this equilibrium to exist is

$$
1 \leq \sqrt{a_{10}}\left(1-\sqrt{\frac{k_{00}}{a_{00}}}\right)+\sqrt{b_{01}}\left(1-\sqrt{\frac{k_{00}}{b_{00}}}\right) .
$$

This condition is more likely to be satisfied the larger $a_{01}$ and $b_{01}$ are and the smaller $k_{00}$ is. See figure 18.

4) Coalition $00-11$ : In this case the policy outcome would be a policy in the set of policies supported by coalition $00-11$ that: gives party 00 at least the same utility that it could obtain from joining either party 01 or party 10 in a coalition, and gives party 11 least the same utility that it could obtain from joining the three party coalition.

Consider any policy $(\widetilde{x}, \widetilde{y})$ that belongs to the set of acceptable policies by the coalition of three parties $01-11-10$. Then for any $(x, y)$ such that $U_{11}(x, y) \geq$ $U_{11}(\widetilde{x}, \widetilde{y})$ we must have one of the three following conditions holding:

i) $U_{01}(x, y) \geq 0$

ii) $U_{10}(x, y) \geq 0$

iii) $U_{01}(x, y)<0$ and $U_{10}(x, y)<0$.

Observe that:

(i) implies that: $U_{00}(x, y)<U_{00}(0, y)$ and $U_{01}(x, y)<U_{01}(0, y)$.

(ii) implies that: $U_{00}(x, y)<U_{00}(x, 0)$ and $U_{10}(x, y)<U_{10}(x, 0)$.

(iii) implies that: $U_{00}(x, y)<U_{00}(0, y)$ and $U_{01}(x, y)<U_{01}(0, y)$; and $U_{00}(x, y)<U_{00}(x, 0)$ and $U_{10}(x, y)<U_{10}(x, 0)$.

Therefore, for any offer from party 00 that might be accepted by party 11 , there is an offer from either party 01 or party 10 that is preferred by party 00 . See figure 17 . 


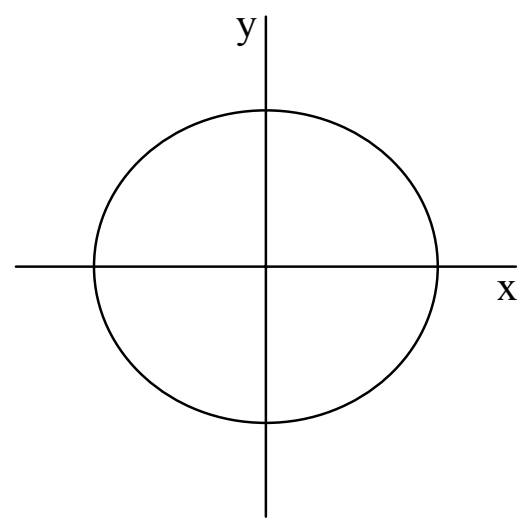

$a_{i}=b_{i}$

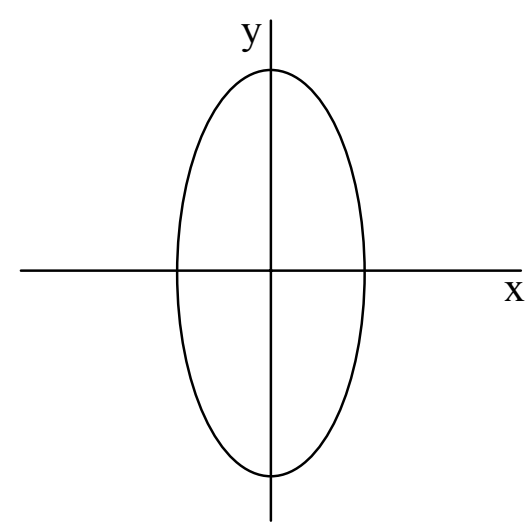

$a_{i}>b_{i}$

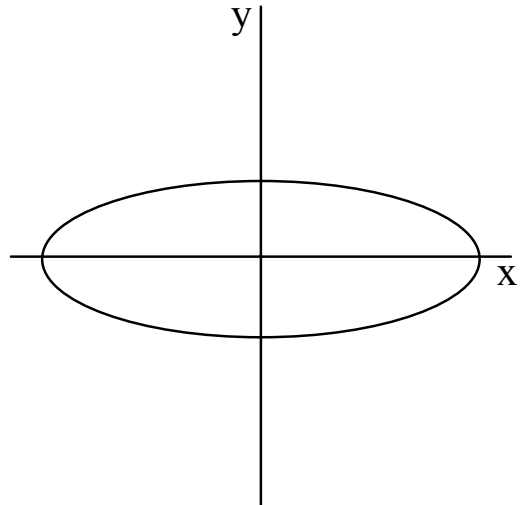

$\mathrm{a}_{\mathrm{i}}<\mathrm{b}_{\mathrm{i}}$

FIGURE 1: Indifference curves.

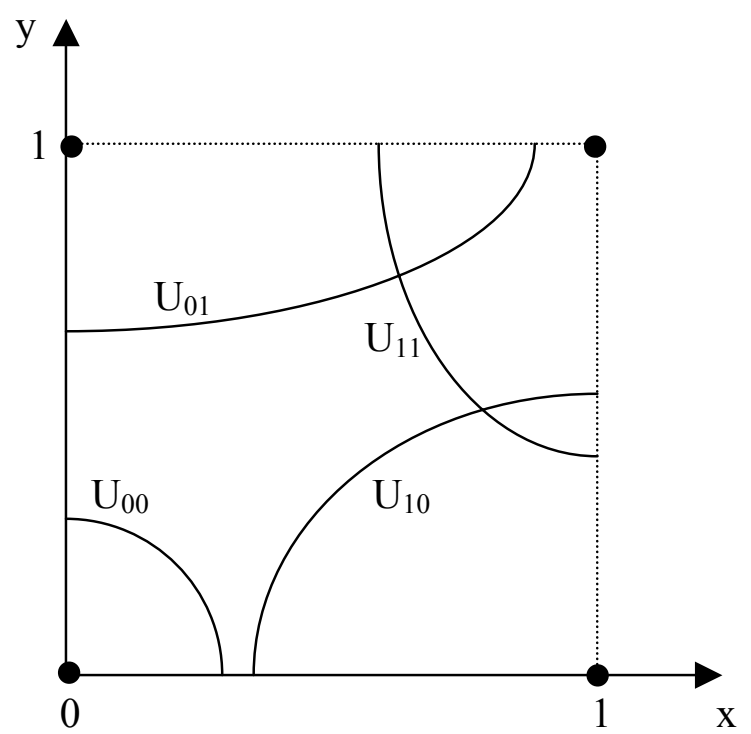

FIGURE 2: Pareto Set and

Individual Rationality constraints. 


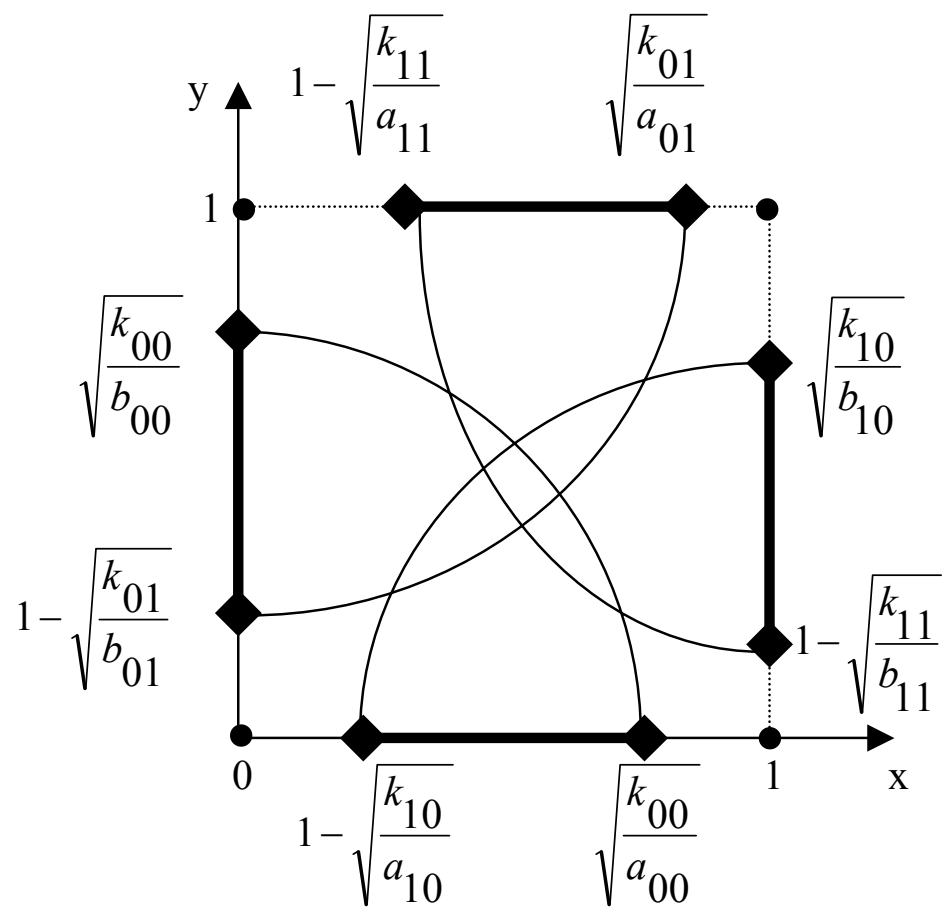

FIGURE 3: Policies acceptable by coalitions of 2 parties that agree on one issue.

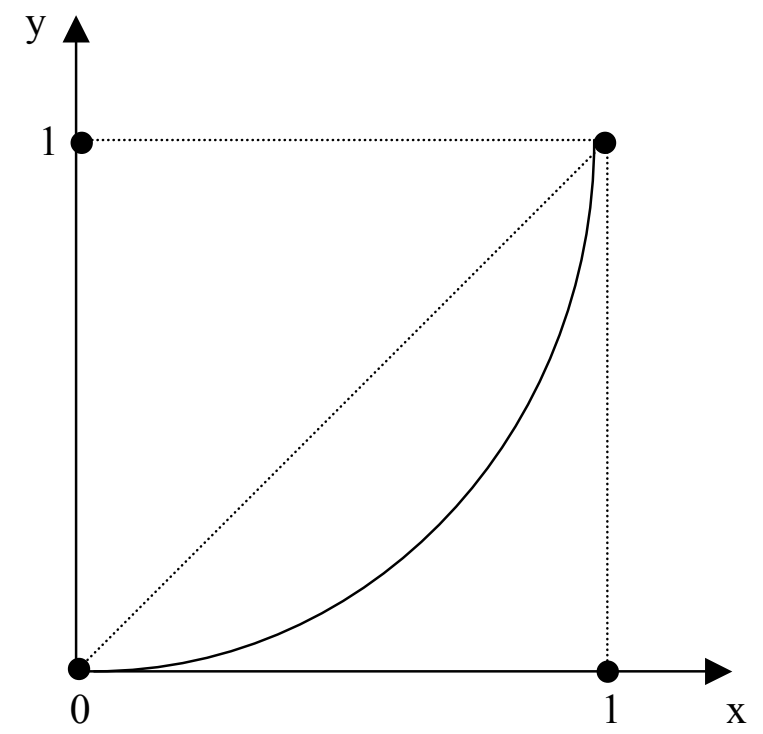

$\mathrm{a}_{00}<\mathrm{a}_{11}$

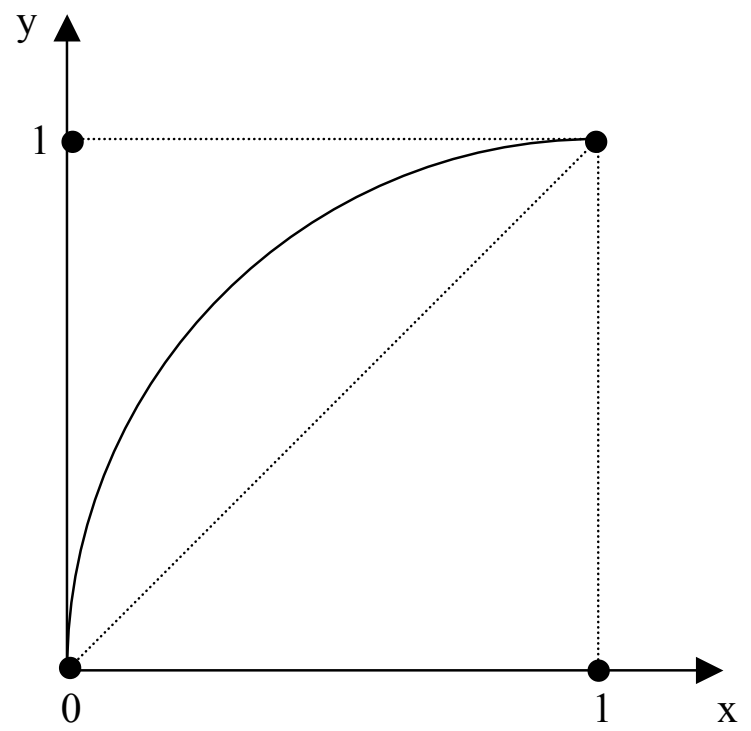

$\mathrm{a}_{00}>\mathrm{a}_{11}$

FIGURE 4: Pareto Optimality for coalition 00-11. 


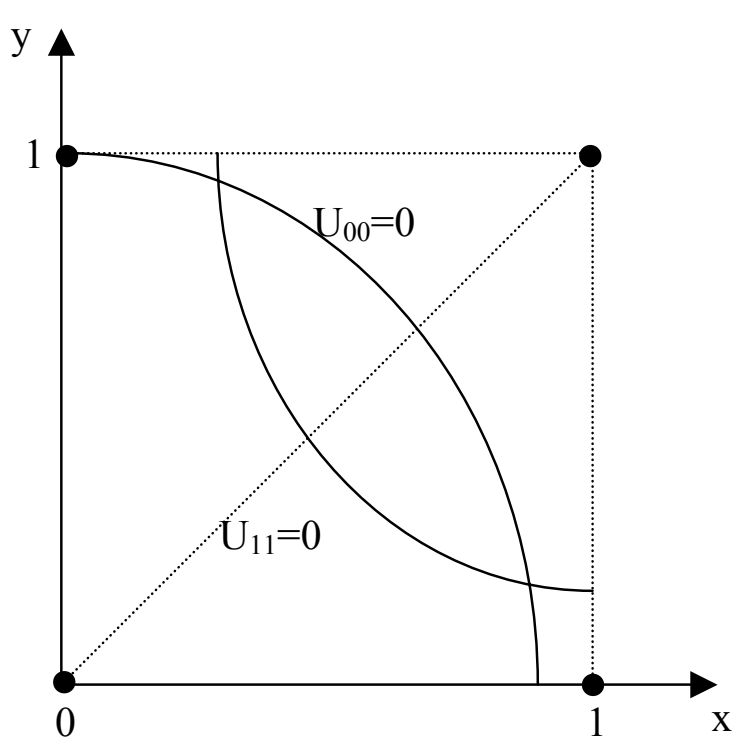

FIGURE 5: Individual rationality constraints for coalition 00-11.

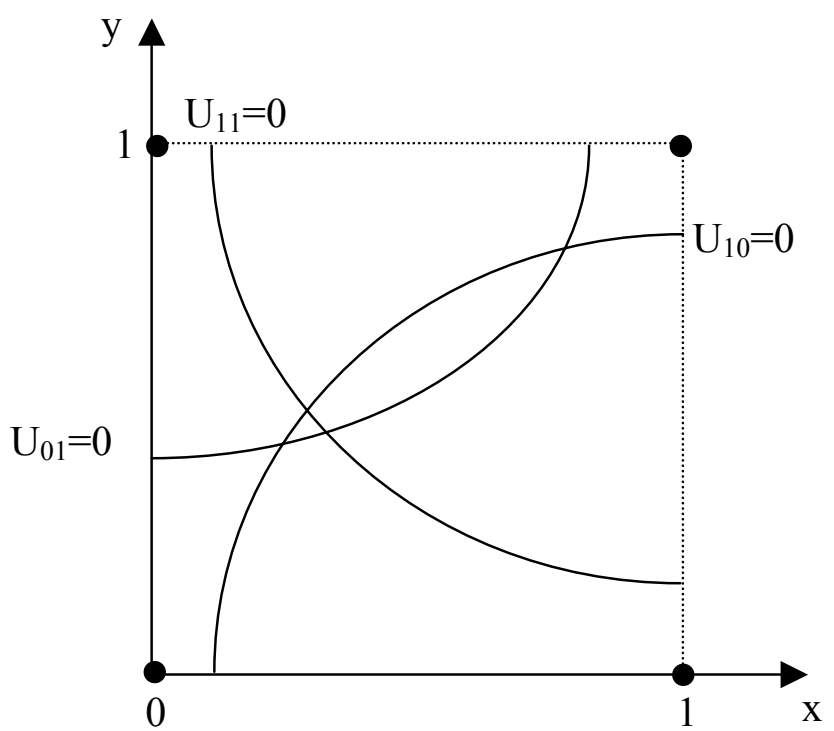

FIGURE 7: Non-empty set of policies acceptable by coalition 01-11-10.

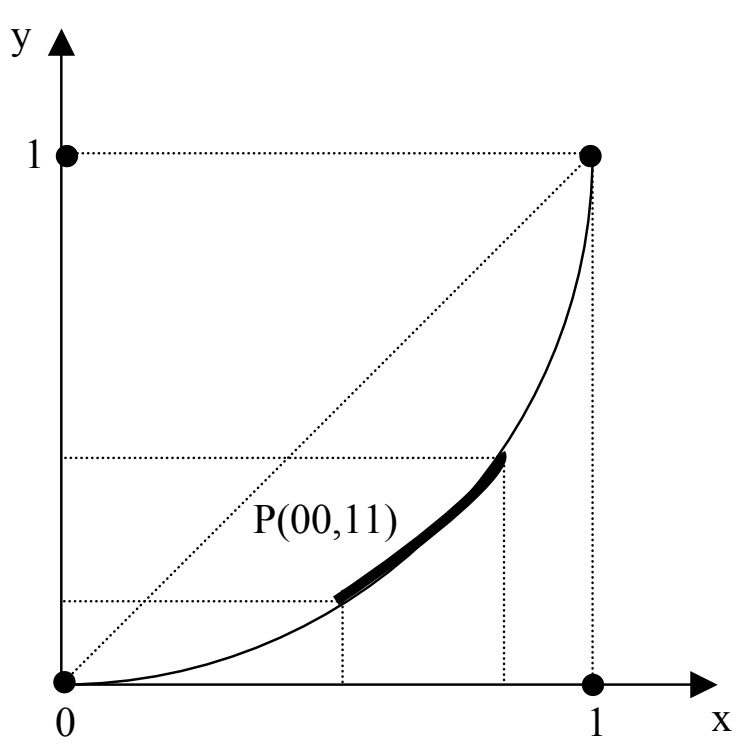

FIGURE 6: Policies acceptable by coalition 00-11.

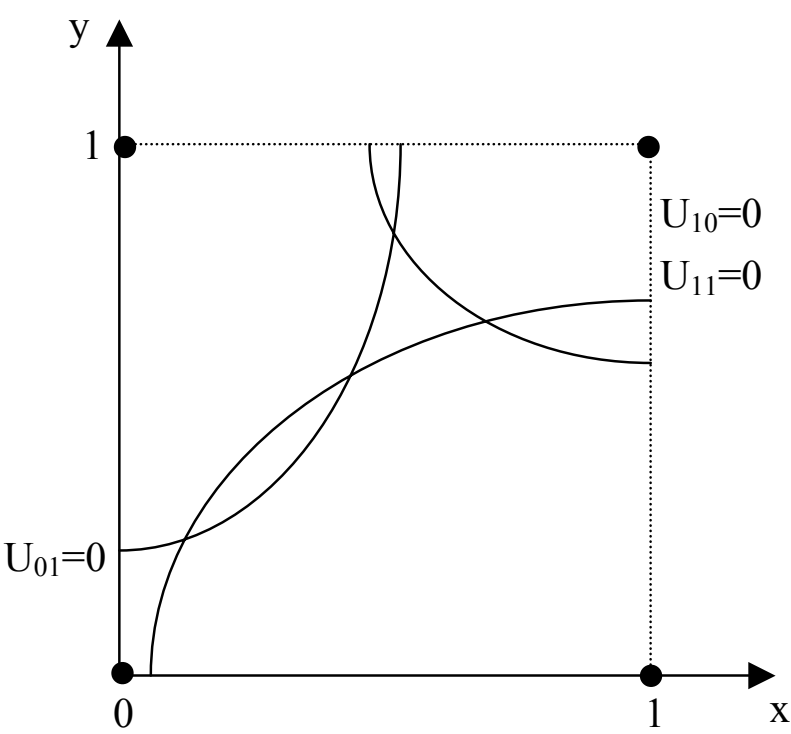

FIGURE 8: Empty set of policies acceptable by coalition 01-11-10. 


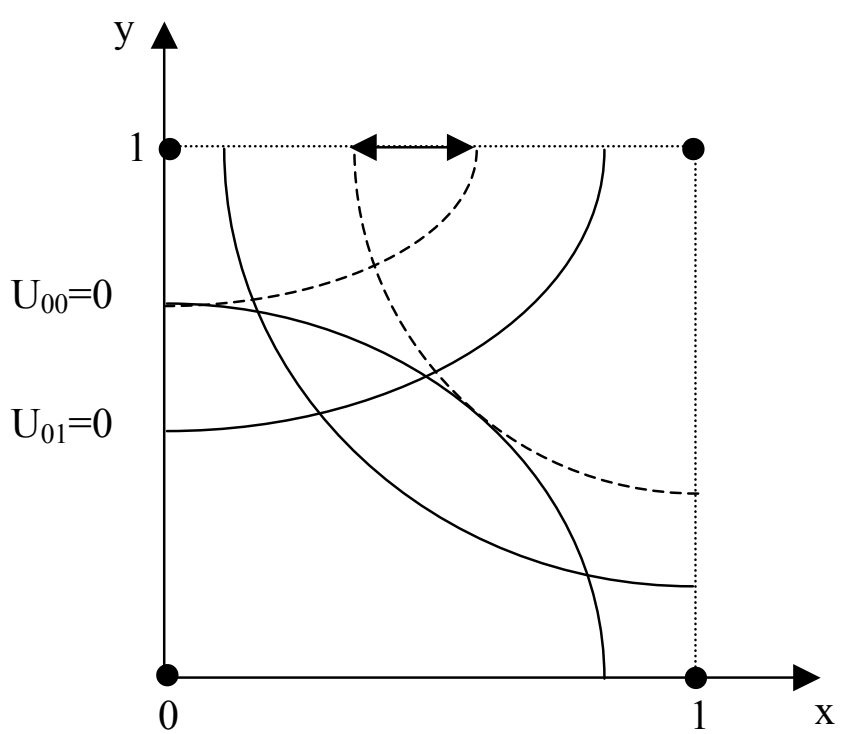

FIGURE 9: Dominated party scenario. Coalition 01-11 in equilibrium.

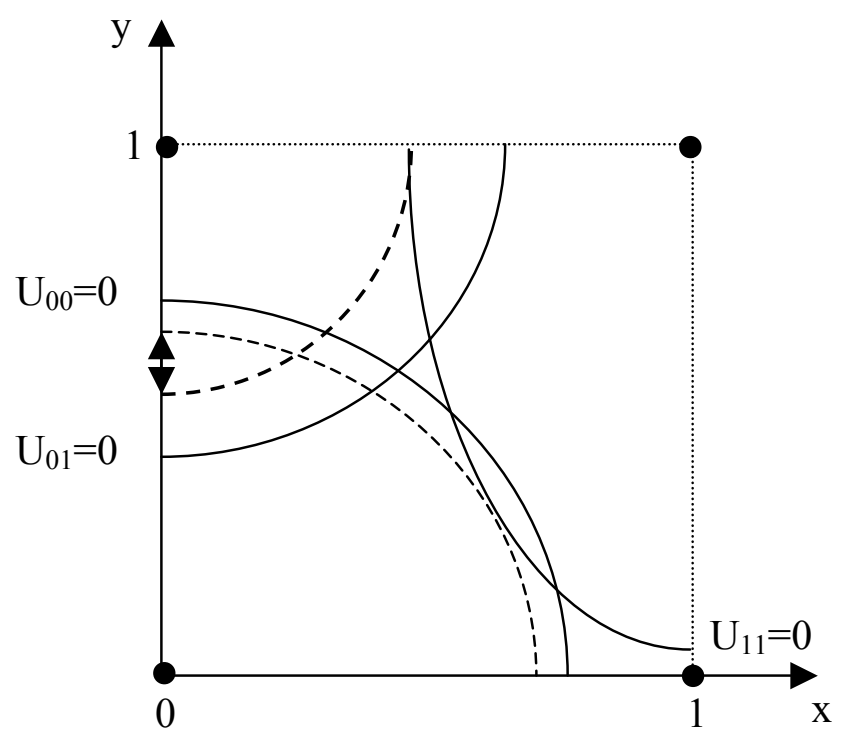

FIGURE 10: Dominated party scenario. Coalition 01-00 in equilibrium.

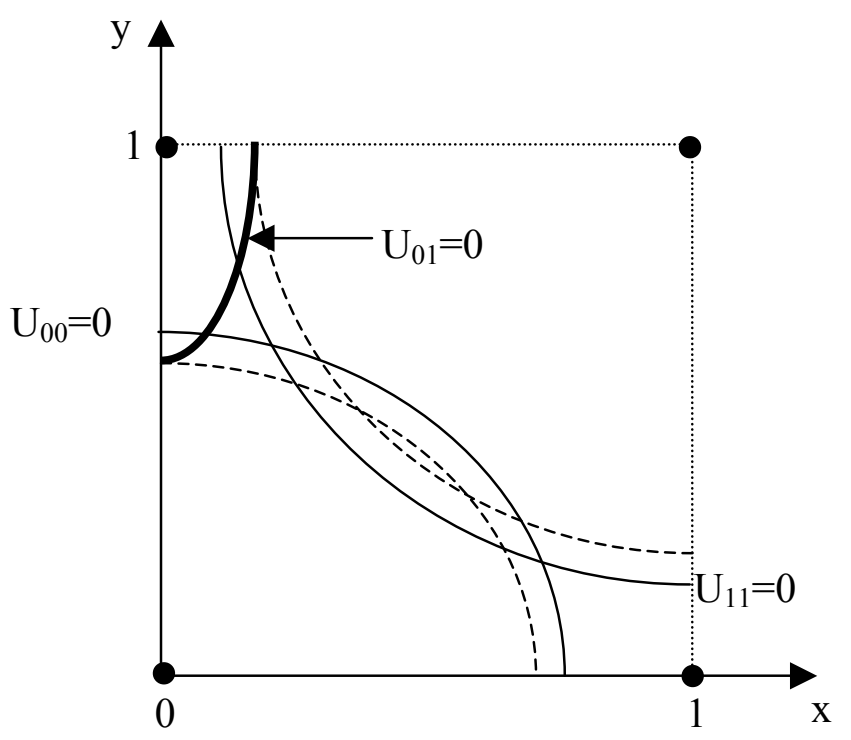

FIGURE 11: Dominated party scenario.

Coalition 00-11 in equilibrium.

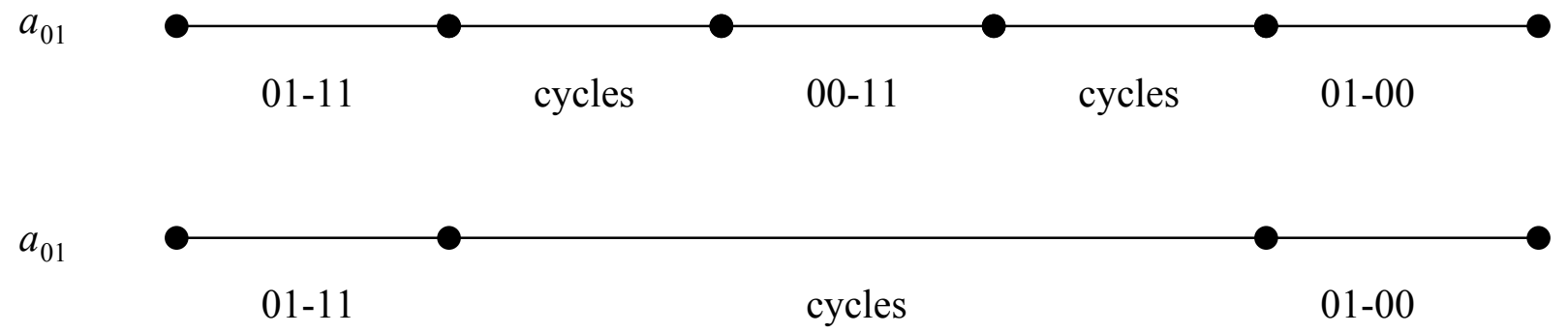

FIGURE 12: Dominated party scenario.

Equilibrium coalitions depending on the value of $\mathrm{a}_{01}$. 


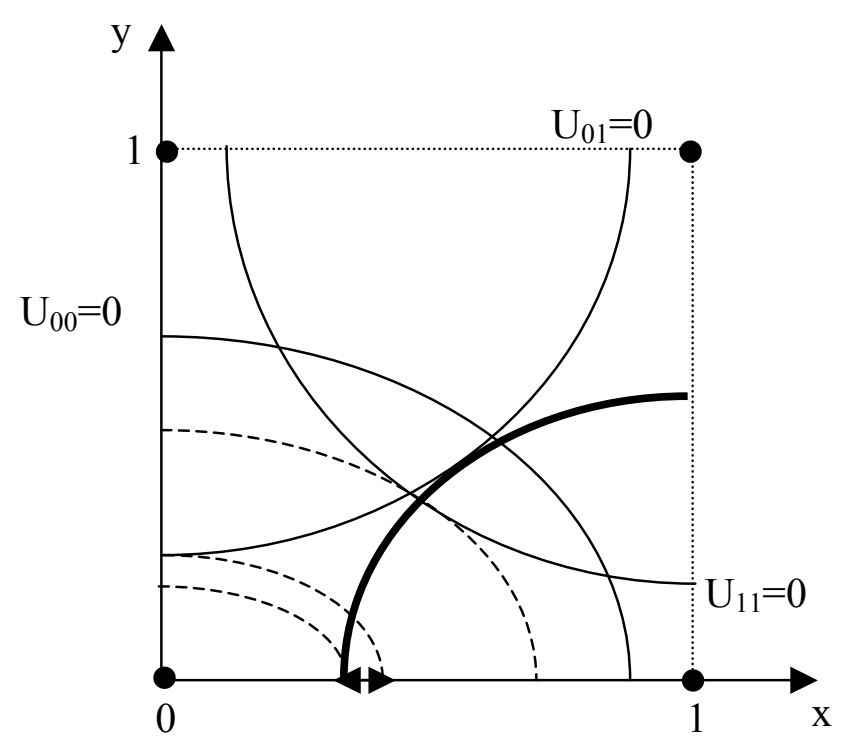

FIGURE 13: Dominant party scenario. Coalition 00-10 in equilibrium. Set of policies determined party 01 threat.

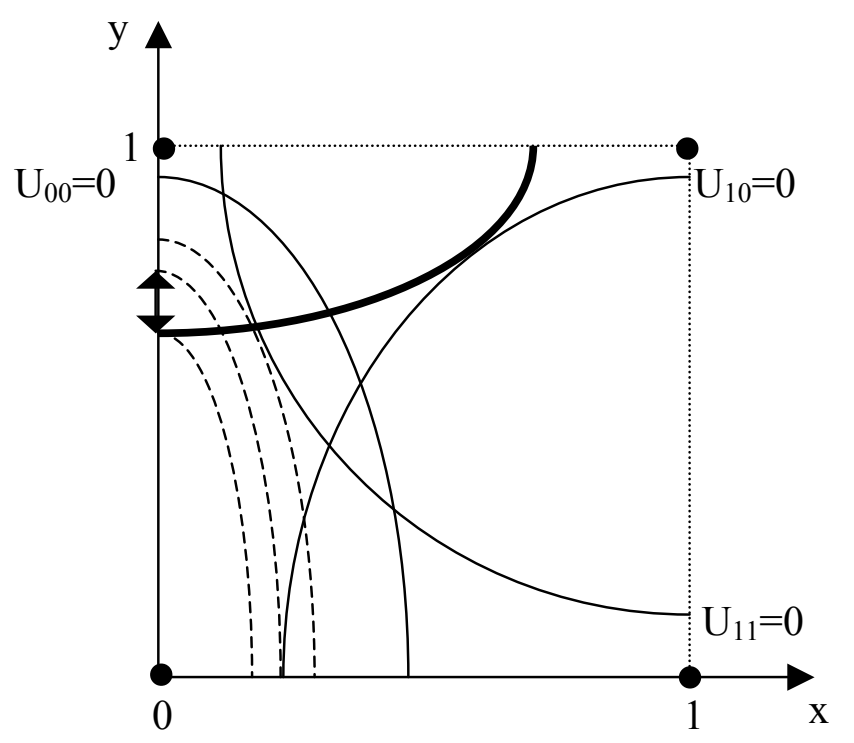

FIGURE 15: Dominant party scenario. Coalition 00-01 in equilibrium. Set of policies determined by party 10 threat.

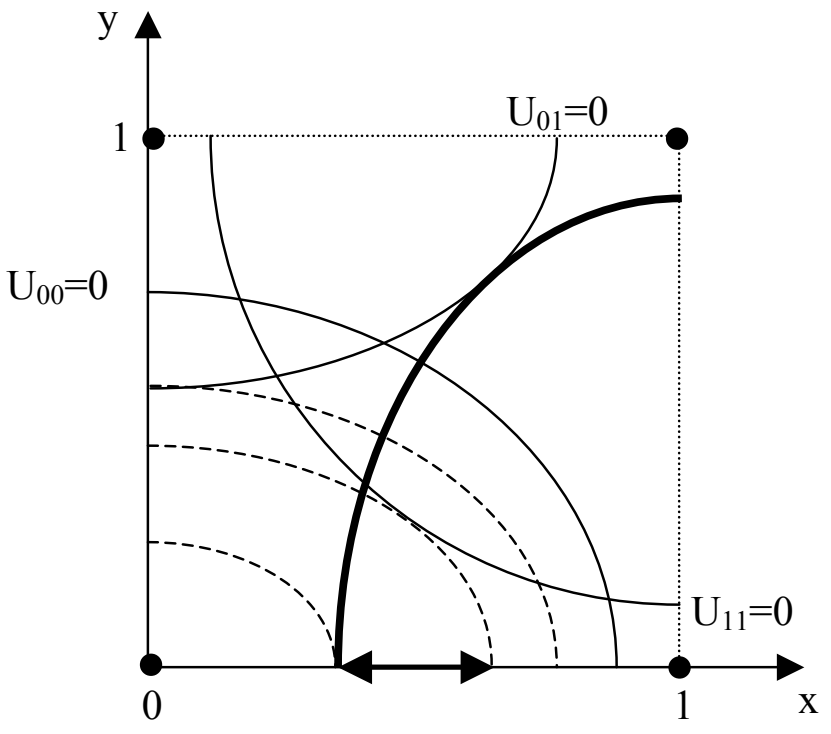

FIGURE 14: Dominant party scenario. Coalition 00-10 in equilibrium. Set of policies determined party 11 threat.

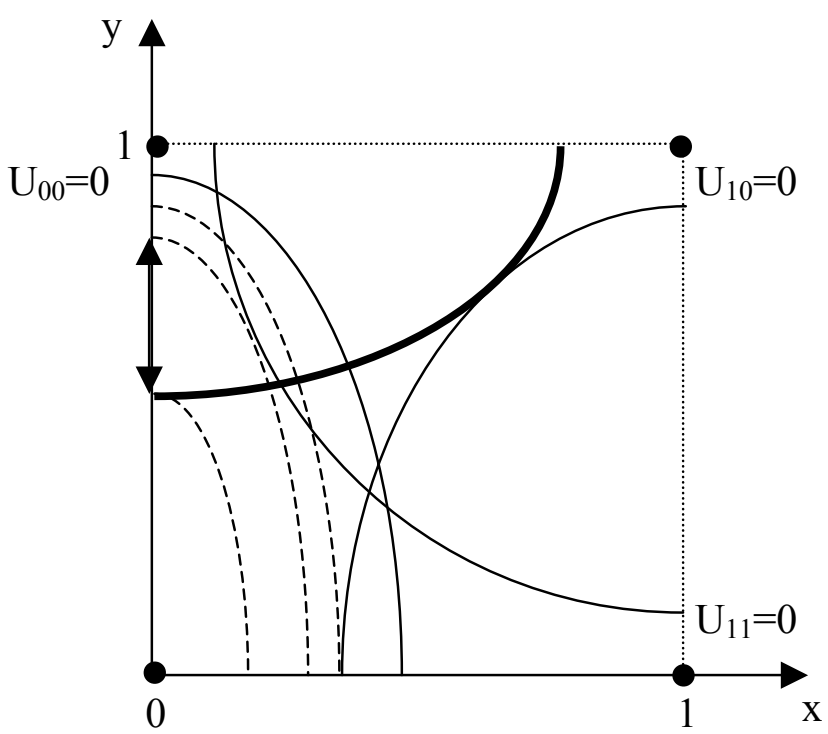

FIGURE 16: Dominant party scenario. Coalition 00-01 in equilibrium. Set of policies determined by party 11 threat. 


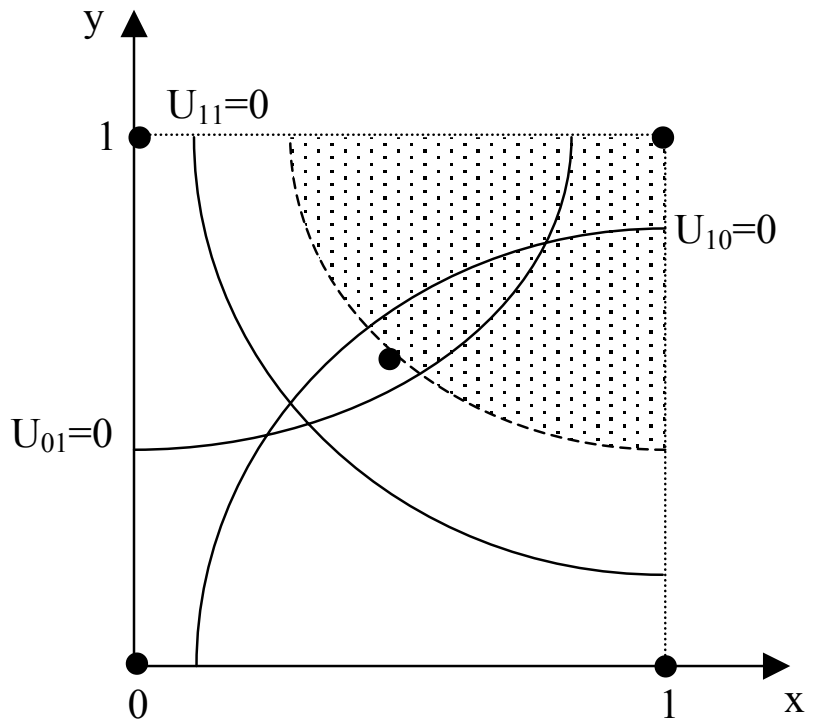

FIGURE 17. Dominant party scenario. Coalition 00-11 cannot be part of an equilibrium.

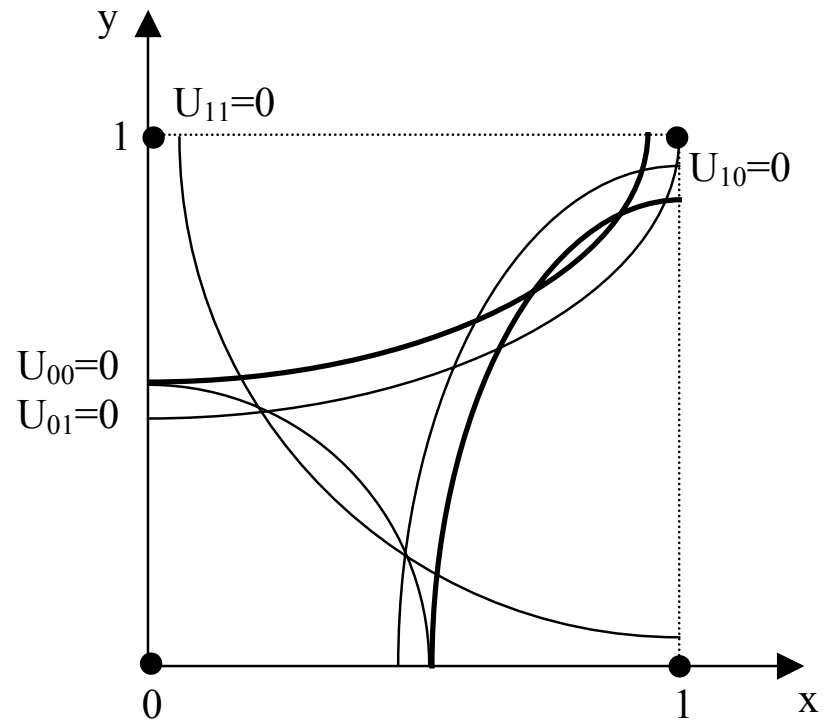

FIGURE 18: Dominant party scenario. Coalition 01-11-10 may be part of an equilibrium.

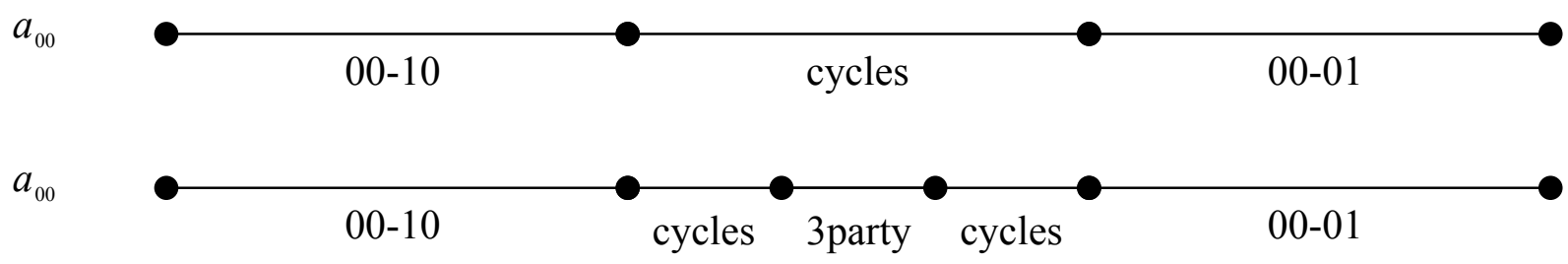

FIGURE 19: Dominant party scenario.

Equilibrium coalitions depending on the value of $\mathrm{a}_{00}$.

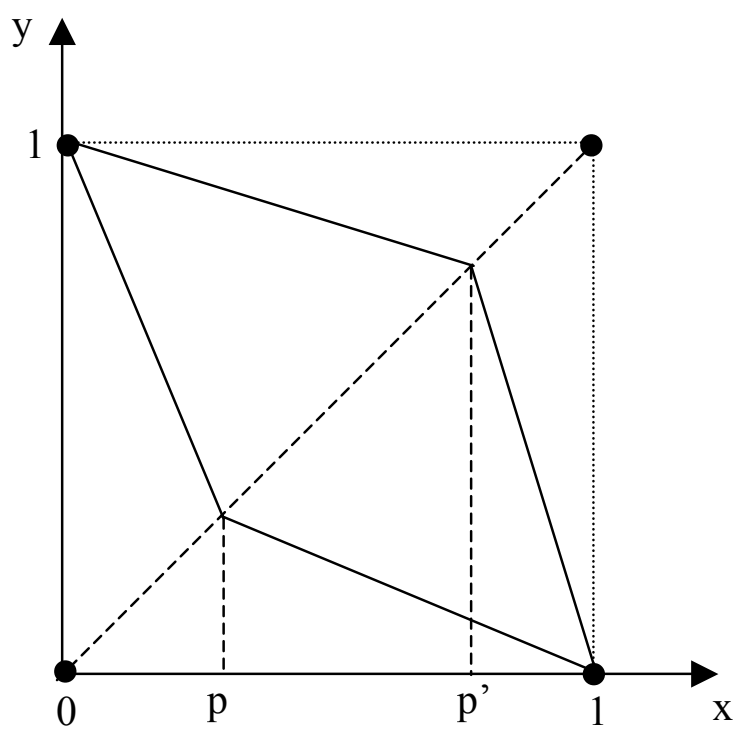

FIGURE 20: $\mathrm{a}_{\mathrm{i}}=\mathrm{a}$ for all $\mathrm{i}$

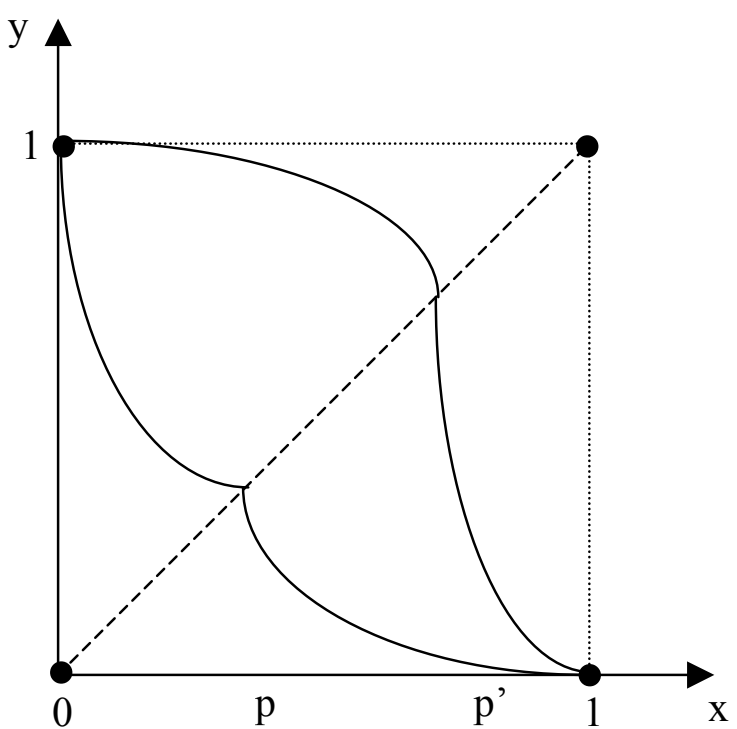

FIGURE 21: $a_{i} \neq a_{j}$ for all $i$ and $j$. 